\title{
Eine Erweiterung der NGB-Mengenlehre als Grundlage der Kategorientheorie *
}

\author{
von \\ Gerhard Os i us (Bremen)
}

\begin{abstract}
We introduce in NBG set theory with urelements a 1-1-correspondence between urelements and certain proper classes (together with further axioms), which enable us to regard these proper classes as "elements" in a new sense. The theory thus obtained is shown to be consistent (using new kinds of inner models) iff a weakening of a standard set theory is consistent. Finally our theory is proposed as a set-theoretical foundation for category theory and its advantages over standard foundations is outlined.
\end{abstract}

\section{§ 0. Einleitung und Utberblick}

Angeregt durch eine Arbeit von A. Oberschelp [5] soll hier eine Erweiterung der Von Neumann-Bernays-Gödel'schen Mengenlehre mit Urelementen (kurz: NBG Theorie), wie sic etwa in Rubin [8] dargestellt ist, betrachtet und als mengentheoretische Grundlage der Kategorientheorie vorgeschlagen werden. Die Grundidee dieser Erweiterung soll schon hier kurz skizziert werden. Wir wollen eine eindeutige Zuordnung zwischen Urelementen und gewissen eigentlichen Klassen herstellen, die es dann gestattet, solche eigentlichen Klassen über die zugeordneten Urelemente wieder als „Elemente” aufzufassen. (Die Idee, eigentliche Klassen und Urelemente in Verbindung zu bringen, geht auf Oberschelp [5] - man vgl. auch [6] - zurück; unsere Theorie weicht allerdings von der aus [5] gänzlich ab.) Es wird daher ein Operator "C" in die NBG-Theorie eingeführt, der jedem Urelement eindeutig eine eigentliche Klasse zuordnet und (aus technischen Gründen) auf den Mengen identisch operiert. Dadurch wird jedem

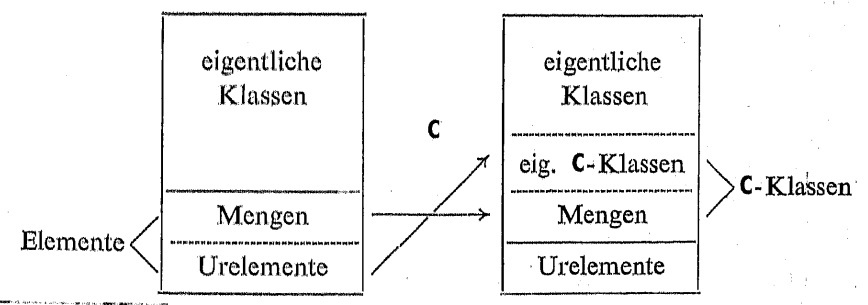

* Neufassung gleichnahmiger Aufzoichnungen an der Universität Bielefeld (1970). 
Element $x$ eine Klasse $\mathbf{C} x$ zugeornet, und die Klassen dieser Art nennen wir C-Klassen (nicht jede Klasse wird C-Klasse sein).

Bezeichnen wir die inverse Operation $\mathbf{C}^{-1}$ kurz mit „e,", so können wir für Klassen $y$ und C-Klassen $x$ die Beziehung e $x \in y$ als , $x$ ist Element von $y$ " (in einem. allgemeineren Sinn) auffassen. Dies entspricht sogar der vom "working mathematician” geübten Praxis, Klassen wieder als Elemente zu verwenden, sofern man dabei die ,innere Struktur” der Klassen „vergißt”, und dieses Vergessen leistet gerade der Operator „„e.

Wir geben jetzt noch einen kurzen Uberblick über unser Vorgehen. In $\S 1$ wird die NBG-Theorie mit Urelementen skizziert, der Begriff des Fundaments von Klassen wird eingeführt, und es werden innere Modelle betrachtet. Dann wird in $\S 2$ die "C-Erweiterung” dargestellt, und die Idee C-Klassen wieder als „Elemente" aufzufassen gibt Anlaß zur modulierten Theorie (die durch Identifikation von C-Klassen $x$ mit den Elementen ex entsteht). Der Wunsch, mit C-Klassen ähnlich umgehen zu können wie mit Mengen, führt zu den C-Klassenbildungsaxiomen. Die so entstandene Theorie nennen wir C-Theorie. Es wird dann eine aufsteigende Familie $\left(\underline{\mathrm{A}}_{a}\right)_{a \in \Omega}$ von $\mathbf{C}$-Klassen definiert, das Abschlußprinzip ${ }_{a \in \Omega} \bigcup_{a} \underline{\mathbf{A}}_{a}$ $=\underline{E}$ (Klasse aller Elemente)" formuliert und seine relative Widerspruchsfreiheit gezeigt. Die weitere Entwicklung der C-Theorie wird noch angedeutet. In $\S 3$ beschäftigen wir uns mit der Frage der Widerspruchsfreïheit der C-Erweiterung. Unser Hauptresultat ist, daß die $\mathbf{C}_{-}$-Theorie genau dann widerspruchsfrei ist, wenn eine modifizierte NBG-Theorie widerspruchsfrei ist. Die modifizierte NBG-Theorie ist hierbei im wesentlichen die reine NBG-Mengenlehre (d.h. ohne Urelemente) mit einem abgeschwächten Ersetzungsprinzip und einer unerreichbaren Kardinalzahl $>\omega$ bzw. mit einem. Universum. Abschließend erläutern wir in $\S 4$ kurz, inwieweit die C-Theorie eine zufriedenstellende mengentheoretische Grundlage der Kategorientheorie sein kann und welche diesbezüglichen Vorteile sie "gegenüber den Standard-Mengentheorien hat.

\section{§ 1. Die NBG-Mengentheorie mit Urelementen}

1.0. Formulierung der Axiome. Die Grundlage unserer Betrachtungen bildet die NBG-Mengenlehre mit Urobjekten, die hier in einer für uns zweckmäßigen Weise skizziert wird. Es handelt sich hierbei um eine Theorie mit Standardformalisierung, die wir jedoch nicht formal darstellen, mit den folgenden drei Grundbegriffen:

$\in$ (Elementbeziehung), zweistelliges Prädikat,

El (Elementprädikat), einstelliges Prädikat,

$\mathrm{Cl}$ (Klassenprädikat), einstelliges Prädikat.

Als logische Zeichnen verwenden wir wie üblich: $\vee$ (es gibt).
Unter den Formeln verstehen wir dann genau die Zeichenreihen, die man aus den sogenannten atomaren Formeln $x \in y, \mathrm{El} x, \mathrm{Cl} x$ unter Verwendung obiger logischer Verknüpfungen auf Standardweise bilden kann.

Die Gleichheit soll hier nicht als logischer Grundbegriff aufgefaßt, sondern nach einem Verfahren von Quine definiert werden durch

$(1.0 .0) \quad x=y: \Leftrightarrow(\mathrm{El} x \Leftrightarrow \mathrm{El} y) \wedge(\mathrm{Cl} x \Leftrightarrow \mathrm{Cl} y) \wedge \bigwedge_{\%}((z \in x \Leftrightarrow z \in y) \wedge(x \in z \Leftrightarrow y \in z))$.

Es gelten dann die charakteristischen Eigenschaften der Gleichheit

$$
x=x \text {. }
$$

(1.0.2) Für jede Formel $\mathfrak{A}(z): x=y \Rightarrow(\mathfrak{H}(x) \Leftrightarrow \mathfrak{U}(y))$.

Die Rahmenaxiome und das Extensionalitätsaxiom lauten:

R1: $x \in y \Rightarrow \mathrm{El} x \wedge \mathrm{Cl} y$,

R2: $\quad$ El $x \Rightarrow \bigvee_{y} x \in y$,

R3: $\mathrm{El} x \vee \mathrm{Cl} x$,

EXT: $\mathrm{Cl} x, y \wedge \bigwedge_{z}(z \in x \Leftrightarrow z \in y) \Rightarrow x=y$.

Der Begriff „El” ist nach R1, 2 durch „E" definierbar. Wir führen jetzt Mengen, Urelemente und eigentliche (proper) Klassen ein.

$$
\begin{aligned}
\operatorname{Mg} x & \Leftrightarrow \mathrm{El} x \wedge \mathrm{Cl} x, \\
\mathrm{Ur} x & \Leftrightarrow \mathrm{El} x \wedge \neg \mathrm{Cl} x, \\
\operatorname{Pcl} x & \Leftrightarrow \neg \mathrm{El} x \wedge \mathrm{Cl} x .
\end{aligned}
$$

Der von uns betrachtete Bereich von Objekten zerfällt also wie folgt:

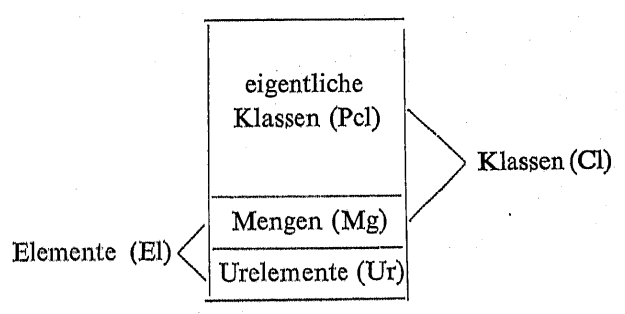

Die Klassenbildung wird gesichert durch das folgende

Klassenkomprehensionsaxiomschema:

KOMP: Für jede Formel $\mathfrak{A}(x)$ im engeren Sinn (d.h. in $\mathfrak{A}(x)$ sind Quantoren auf Elemente beschränkt: $\left.\bigwedge_{\mathrm{El} z}, \bigvee_{\mathrm{El} z}\right)$ ist die Formel,$\bigvee_{\mathrm{Cl} Y \mathrm{Bl} x}(x \in y \Leftrightarrow \mathfrak{U}(x))^{*}$ ein Axiom. 
Es ist bekannt (vgl. Gödel), daß man dieses Axiomschema unter Verwendung noch folgender Mengenbildungsaxiome äquivalent durch eine endliche Zah1 von Axiomen ersetzen kann.

Nach einem Vorschlag von Quine fordert man gelegentlich auch ein stärkeres Komprehensionsschema KOMPS, indem man in KOMP auf die Nebenbedingungen an $\mathfrak{H}(x)$ (daß gebundene Variablen auf Elemente relativiert sind) verzichtet. Die so verschärfte Theorie werden wir nur am Rande betrachten und sie kurz als $N B G Q$-Theorie bezeichnen.

Nach KOMP und EXT läßt sich für jede Formel $\mathfrak{2}(x)$ im engeren Sinne im Bezug auf die Variable $x$ eine Klasse $\{x \mid \mathfrak{Q}(x)\}$ definieren durch:

$$
\bigwedge_{\mathrm{El} z} z \in\{x \mid \mathfrak{Q}(x)\} \Leftrightarrow \mathfrak{Q}(z) \text {. }
$$

Jetzt werden unter Verwendung von Standard-Definitionen die (nicht voneinander unabhängigen) Mengenbildungsaxiome formuliert.

\section{Axiom der leeren Menge:}

LM: $\mathrm{Mg} \varnothing$.

\section{Zweiermengenaxiom:}

ZM: $\quad$ El $x, y \Rightarrow \operatorname{Mg}\{x, y\}$.

Vereinigungsmengenaxiom:

\section{VM: $\quad \operatorname{Mg} x \Rightarrow \operatorname{Mg} \cup x$.}

Potenzmengenaxiom:

\section{PM: $\quad \operatorname{Mg} x \Rightarrow \operatorname{Mg} P x$.}

Aussonderungsaxiom:

AUS: $\operatorname{Mg} x \wedge \mathrm{Cl} y \wedge y \subset x \Rightarrow \operatorname{Mg} y$.

Unendlichkeitsaxiom:

\section{UN: $\quad \mathbf{M g} \omega$.}

Unter Verwendung des Begriffs des geordneten Paares von Elementen und des Abbildungsbegriffs läßt sich das Aussonderungsprinzip nach Fraenkel verschärfen zum

\section{Ersetzungsaxiom}

ERS: $f: x \rightarrow y$ Abbildung $\wedge \operatorname{Mg} x \Rightarrow \operatorname{Mg} f[x]$.

Die so beschriebene Theorie soll im folgenden als NBG-Theorie bezeichnet werden, obwohl noch einige „Axiome” fehlen, die wir hier als „Prinzipien” noch notieren und deren Gebrauch jeweils notiert wird (z.B. in (1.1.14)).

\section{Fundierungsprinzip:}

FUND: $\quad \operatorname{Cl} x \wedge x \neq \varnothing \wedge \bigwedge_{y \in x} \operatorname{Mg} y \Rightarrow \bigvee_{y \in x} y \cap x=\varnothing$.

\section{Auswahlprinzip für Mengen}

AM: $\quad \operatorname{Mg} x \wedge \bigwedge_{y \in x}(\operatorname{Mg} y \wedge y \neq \varnothing) \Rightarrow \bigvee_{f: x \rightarrow \cup x \text { Abb }} \bigwedge_{y \in x} f(y) \in y$.

Auf die Darstellung weiterer Einzelheiten der NBG-Theorie können wir aufgrund der zahlreichen Literatur verzichten und die wichtigsten Resultate im folgenden voraussetzen.

Für den späteren Gebrauch treffen wir noch einige Definitionen

$$
\begin{aligned}
& \underline{E}:=\{x \mid \operatorname{El} x\}, \\
& \underline{U}:=\{x \mid \operatorname{Ur} x\}, \\
& \underline{\mathrm{V}}:=\{x \mid \operatorname{Mg} x\} .
\end{aligned}
$$

Fordert man "U $=\varnothing$ " (was weder beweis - noch widerlegbar ist) als Axiom, so erhält man die (übliche) reine. NBG-Theorie.

1.1. Der Begriff des Fundaments. Ist $K$ eine beliebige Klasse, so intereșsieren wir uns für die Klasse der Urelemente, die zum „Aufbau” v̧on $K$ benötigt werden. Zunächst definieren wir die transitive Hülle von $K$ als Klasse aller Elemente aus denen. $K$,aufgebaut" ist.

$$
\operatorname{Tr} K:=\left\{x \mid \bigvee_{n \in \omega} \bigvee_{\left(x_{0}, \ldots, x_{n}\right) \in \mathrm{E}^{n+1}} x=x_{0} \in x_{1} \in \ldots \in x_{n} \in K\right\}
$$

Für eine Menge $L$ läßt sich eine andere Beschreibung der transitiven Hülle geben. Hieral definieren wir rekursiv die $n$-fache Vereinigung:

$$
\bigcup^{0} L:=L, \bigwedge_{n \in \omega} \bigcup^{n+1} L:=\bigcup \bigcup^{n} L
$$

Es gilt dann

$$
\begin{gathered}
\operatorname{Mg} K \Rightarrow \bigwedge_{n \in \omega} U^{n} K=\left\{x \mid \underset{\left(x_{0}, \ldots, x_{n}\right) \in \underline{E}^{n+1}}{\bigvee} x=x_{0} \in x_{1} \in \ldots \in x_{n} \in K\right\}, \\
\operatorname{Mg} K \Rightarrow \operatorname{Tr} K=\bigcup_{n \in \omega} U^{n} K, \\
\operatorname{Mg} K \Rightarrow \operatorname{MgTr} K .
\end{gathered}
$$

Das Fundament von $K$ wird nun als die Klasse der zum Aufbau von $K$ benötigten Urelemente definiert:

$$
\mathrm{Fd} K:=\underline{\mathrm{U}} \cap \operatorname{Tr} K .
$$

Ist nun umgekehrt $U \subset U$ eine Klasse von Urelementen, so suchen wir nach allen Klassen, deren Fundament in $U$ liegt. Definieren wir

$$
\begin{aligned}
& \underline{\mathrm{V}}(U):=\underline{\mathrm{V}}_{U}:=\{x \mid \mathrm{Mg} x \wedge \mathrm{Fd} x \subset U\}, \\
& \underline{\mathrm{E}}(U):=\underline{\mathrm{E}}_{U}:=U \cup \underline{\mathrm{V}}(U)
\end{aligned}
$$


so gilt die wichtige Beziehung

$$
\text { (1.1.8) } \quad K \subset \underline{\mathrm{E}}(U) \Leftrightarrow \mathrm{Fd} K \subset U \text {. }
$$

Beweis (1.1.8):

m,: Sei $x \in \mathrm{Fd} K$, also $x=x_{0} \in x_{1} \in \ldots \in x_{n} \in K$ und $x \in \underline{\mathrm{U}}$. Für $n=0$ ist $x \in K \cap \underline{\mathrm{U}} \subset \underline{\mathrm{E}}(U) \cap \underline{\mathrm{U}}=U$ und für $n>0$ ist $x_{n} \in K \cap \underline{\mathrm{V}} \subset \underline{\mathrm{V}}(U)$ und $x \in \mathrm{Fd} x_{n} \subset U$. Also $\operatorname{Fd} \bar{K} \subset \bar{U}$.

"Е: Es gilt $K \cap \underline{U} \subset \mathrm{Fd} K \subset U$ und für Mengen $x \in K$ ist $\mathrm{Fd} x \subset \mathrm{Fd} K \subset U$, also $x \in \underline{\mathrm{V}}(U)$. Also $K \bar{\subset} E(U)$.

Speziell für $K=\underline{\mathrm{E}}(U)$ erhält man aus (1.1.8)

$$
\operatorname{Fd} \underline{E}(U)=U \text {. }
$$

Die Klassen $\underline{E}(U)$ lassen sich auch ohne Verwendung des Fundamentbegriffes kennzeichnen, denn für eine beliebige Klasse $K$ gilt:

$$
P K=K \cap \underline{\mathrm{V}} \Leftrightarrow K=\underline{\mathrm{E}}(\mathrm{Fd} K) .
$$

Insbesondere gilt also für $U \subset \underline{U}$

$$
P \underline{\mathrm{E}}(U)=\underline{\mathrm{V}}(U) .
$$

Beweis (1.1.10):

$\Longrightarrow "$ Man zeigt $\mathrm{Fd} K \subset K$ und erhält mit (1.1.8) $K \subset \underline{\mathrm{E}}(\mathrm{Fd} K)$ und $\underline{\mathrm{E}}(\mathrm{Fd} K) \subset K$ nach Vor.

„Æ" Anwendung von (1.1.8) auf Mengen $K$.

Beweis (1.1.11): Anwendung von (1.1.9) und (1.1.10) auf $K=\mathrm{E}(U)$.

Bevor wir im nächsten Abschnitt die wichtigsten Eigenschaften der Klassen $\mathbf{E}(U)$ (normales inneres Modell zu sein) entdecken, geben wir (unter Verwendung des Fundierungsprinzips) noch eine weitere „rekursive” Charakterisierung dieser Klassen.

Für eine Menge $L$ definieren wir die Konstruktionsabbildung $\underline{K}_{L}: \Omega \rightarrow \underline{V}$ rekursiv durch:

$$
\bigwedge_{a \in \Omega} \underline{K}_{L}(a):=\bigcup_{b<a} L \cup P \underline{K}_{L}(b) .
$$

Für $U \subset \underline{U}$ erhält man durch Induktion sofort (falls $U$ Menge ist):

$$
a \in \Omega \Rightarrow \underline{\mathrm{K}}_{U}(a) \subset \underline{\mathrm{E}}(U) \text {. }
$$

Weiter kann man zeigen (worauf wir hier verzichten):

$$
(1.1 .14)^{\mathrm{FUND}}
$$

$$
\bigcup_{a \in \Omega} \underline{\mathrm{K}}_{U}(a)=\underline{\mathrm{E}}(U) \text {. }
$$

1.2. Innere Modelle. Für spätere $Z$ wecke werden jetzt innere Modelle eingeführt (die nicht genau mit denen von Shepherdson [9] übereinstimmen).
Im folgenden sei $K$ eine fest gewählte Klasse. Dann kann man die Grundbegriffe der NBG-Theorie über dem Bereich der Elemente und Teilklassen von $K$ wie folgt „interpretieren":

$$
\begin{aligned}
\mathrm{Ob}_{K} x & : \Leftrightarrow x \in K \vee(\mathrm{Cl} x \wedge x \subset K), \\
x \in_{\mathrm{K}} y & \Leftrightarrow x \in y \wedge y \subset K, \\
\mathrm{El}_{\mathrm{K}} x & \Leftrightarrow x \in K \\
\mathrm{Cl}_{\mathrm{K}} x & : \Leftrightarrow \mathrm{Cl} x \wedge x \subset K .
\end{aligned}
$$

Rekursiv über den Aufbau läßt sich dann jeder NBG-Formel $\mathfrak{Q}$ ihre Relativierung $2 r_{K}$ auf $K$ zuordnen $(\vee, \Rightarrow, \Leftrightarrow, \bigvee$ seien wie üblich definiert):

$$
\begin{aligned}
(x \in y)_{\mathrm{K}} & : \equiv x \epsilon_{\mathrm{K}} y, \\
(\mathrm{El} x)_{\mathrm{K}} & : \equiv \mathrm{El}_{\mathrm{K}} x, \\
(\mathrm{Cl} x)_{\mathrm{K}} & : \equiv \mathrm{Cl}_{\mathrm{K}} x, \\
(\neg A)_{\mathrm{K}} & : \equiv \neg A_{K}, \\
(A \wedge B)_{\mathrm{K}} & : \equiv A_{\mathrm{K}} \wedge B_{K}, \\
\left(\bigwedge_{x} A\right)_{K}: & \equiv \bigwedge_{\mathrm{ob}_{\mathbb{K}} x} A_{\mathrm{K}} .
\end{aligned}
$$

Zunächst stellen wir fest, daß bei der durch (1.2.0)-(1.2.3) gegebenen Interpretation der NBG-Grundbegriffe die Rahmenaxiome R1-R3 getten, d.h. ihre Relativierungen $\mathbf{R 1}_{K}-\mathbf{R} \mathbf{3}_{K}$ gelten:

$$
\begin{aligned}
\mathrm{Ob}_{\mathrm{K}} x, y \wedge x \epsilon_{\mathrm{K}} y & \Rightarrow \mathrm{El}_{\mathrm{K}} x \wedge \mathrm{Cl}_{\mathrm{K}} y, \\
\mathrm{Ob}_{\mathrm{K}} x \wedge \mathrm{El} x & \Rightarrow \bigvee_{\mathrm{O}_{\mathrm{K}} y} x \in_{\mathrm{K}} y, \\
\mathrm{Ob}_{\mathrm{K}} x & \Rightarrow \mathrm{El}_{\mathrm{K}} x \vee \mathrm{Cl}_{\mathrm{K}} x .
\end{aligned}
$$

Die durch (1.0.0) gegebene Gleichheit liefert bei der Interpretation über $K$ wieder die Gleichheit, d.h.

$$
\text { (1.2.8) } \quad \mathrm{Ob}_{\mathrm{K}} x, y \Rightarrow\left((x=y)_{\mathrm{K}} \Leftrightarrow x=y\right) \text {. }
$$

Hieraus folgt die Gültigkeit des Extensionalitätsaxioms und des Klassenkomprehensionsaxiomschemas bei unserer Interpretation über $K$ :

$$
\mathrm{Cl}_{\mathrm{K}} x, y \wedge \bigwedge_{\mathrm{El}_{\mathrm{K}^{z}}}\left(z \in_{\mathrm{K}} x \Leftrightarrow z \in_{\mathrm{K}} y\right) \Rightarrow x=y .
$$

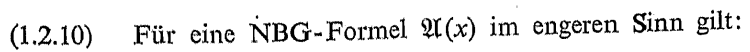

$$
\mathrm{Cl}_{K} K \cap\left\{x \mid \mathfrak{T}_{K}(x)\right\}, \quad \bigwedge_{\mathbf{E}_{\mathbb{K}^{z}}}\left(z \in_{K} K \cap\left\{x \mid \mathfrak{N}_{K}(x)\right\} \Leftrightarrow \mathfrak{A}_{K}(z)\right) .
$$


Man kann nun jede Definition der NBG-Theorie, die nur auf der Gültigkeit der Axiome R1-R3, EXT, KOMP basiert, durch die „Interpretation” (1.2.0)-(1.2.3) auf $K$ relativieren (vgl. Gödel [1], Shepherdson [9]). Die Relativierung eines Begriffs wird durch den Index „K" bezeichnet. Aus (1.2.10) erhält man z. B. die relativierte Klassenkomprehension:

$$
\text { (1.2.11) } \quad\left\{x \mid \mathfrak{A}_{K}(x)\right\}_{K}=K \cap\left\{x \mid \mathfrak{Q T}_{K}(x)\right\} .
$$

Begriffe, die auf den $K$-Objekten mit ihrem relativierten Begriff übereins timmen heißen auch absolut bzgl. $K$ (vgl. [1], [9]).

Absolute Begriffe sind z. B. die Elementrelation (vgl. (1.2.1)), die Gleichheit (vgl. (1.2.8)). Wir werden die Absolutheit einiger elementarer Begriffe stillschweigend benutzen, ohne sie explizit zu erwähnen.

Die Begriffe Klasse, Menge, Urelement sind im allgemeinen nicht absolut:

$$
\begin{array}{rlrl}
\mathrm{Ur}_{K} x & \Leftrightarrow x \in K \wedge x \notin P K, & \underline{\mathrm{U}}_{K}=K \backslash P K, \\
\operatorname{Mg}_{K} x \Leftrightarrow x \in K \wedge x \in P K, & \underline{\mathrm{V}}_{K}=K \cap P K .
\end{array}
$$

Es erhebt sich jetzt die Frage, ob auch die Relativierungen aller Mengenbildungsaxiome gelten bzw. unter welchen Voraussetzungen an $K$ dies der Fall ist. Wir geben die Resultate ohne Beweis an:

$$
\begin{aligned}
& \mathbf{L M}_{K} \Leftrightarrow \varnothing \in K, \\
& \mathbf{Z M}_{K} \Leftrightarrow \bigwedge_{x, y \in \mathbb{K}}\{x, y\} \in K, \\
& \mathbf{V M}_{K} \Leftrightarrow \bigwedge_{x \in K \cap P K \cap P P X} U x \in K, \\
& \mathbf{P M}_{K} \Leftrightarrow \bigwedge_{x \in \mathcal{K} \cap P K} K \cap P x \in K, \\
& \mathbf{A U S}_{K} \Leftrightarrow \bigwedge_{x \in K \cap P K} P x \subset K, \\
& \mathbf{U N}_{K} \Leftrightarrow \omega \in K \cap P K, \\
& \mathbf{Z M}_{K} \Rightarrow\left(\mathbf{E R S}_{K} \Leftrightarrow \bigwedge_{x \in K \cap P K} \bigwedge_{f: x \rightarrow K} f[x] \in K\right) .
\end{aligned}
$$

Bezüglich der Gültigkeit des relativierten Fundierungs- und Auswahlprinzips bemerken wir:

$$
\begin{array}{cc}
(1.2 .21) & \mathbf{F U N D} \Rightarrow \mathbf{F U N D}_{K}, \\
(1.2 .22) & \mathbf{Z M}_{K} \wedge \cdot \mathbf{A M} \Rightarrow \mathbf{A M}_{K} .
\end{array}
$$

$K$ soll jetzt ein inneres Modell heißen, wenn die Relativierungen aller NBGAxiome gelten, d.h. (unter Berücksichtigung von (1.2.5)-(1.2.10):

(1.2.23) $K$ heißt inneres $(N B G-)$ Modell $: \Leftrightarrow$

$(\mathbf{L M} \wedge \mathbf{Z M} \wedge \mathbf{V M} \wedge \mathbf{P M} \wedge \mathbf{A U S} \wedge \mathbf{U N} \wedge \mathbf{E R S})_{\mathbf{K}}$.
Mit (1.2.14)-(1.2.20) erhält man:

(1.2.24) $K$ inneres Modell $\Leftrightarrow \omega \in K \cap P K \wedge$

$$
\left.\wedge \wedge_{x \in K \cap P K}\left((x \subset P K \Rightarrow U x \in K) \wedge P x \in K \cap P K \wedge \bigwedge_{f: x \rightarrow K} f[x] \in K\right)\right) \text {. }
$$

Innere Modelle, bei denen der Begriff Urelement absolut ist, nennen wir normal (sie entsprechen den „super-complete models” aus [9]).

(1.2.25) $K$ normales inneres Modelt $: \Leftrightarrow K$ inneres Modell $\wedge \underline{U}_{K} \subset \underline{\mathrm{U}}$.

Für normale innere M.odelle sind fast alle Begriffe absolut. Als Beispiele für normale innere Modelle geben wir an:

$$
\begin{aligned}
& P K=K \cap \underline{\mathrm{V}} \Rightarrow K \text { normales inneres Modell, } \\
& U \subset \underline{\mathrm{U}} \Rightarrow \underline{\mathrm{E}}(U) \text { normales inneres Modell, } \\
& U \subset \underline{\mathrm{U}} \Rightarrow \bigcup_{a \in \Omega} \underline{\mathrm{K}}_{U}(d) \text { normales inneres Modell . }
\end{aligned}
$$

Man beachte, daß diese inneren Modelle eigentliche Klassen sind. Innere Modelle, die Mengen sind, wollen wir klein nennen. Sie entsprechen bei Verwendung des Auswahlaxioms AM unerreichbaren Mengen, die $\omega$ als Element enthalten (vgl. Tarski [11], Kühnrich [2]).

\section{§ 2. Die C-Erweiterung der NBG-Theorie (C-Theorie)}

2.0. Der neue Grundbegriff $C$. Wir erweitern jetzt die NBG-Theorie um eine Konstante $\mathbf{C}$ (Klassenfunktion) und nennen die Formeln der Erweiterung C-Formeln. Als neue Axiome fordern wir zunächst die Rahmenaxiome R4-7:

\section{R4: $\quad \mathrm{ClC} \wedge \mathrm{C} \subset \underline{\mathrm{E}} \times \underline{\mathrm{E}}$.}

Die Relation $\mathbf{C}$ läßt sich als Operator interpretieren, der jedem Element $x$ die Klasse $\mathbf{C}_{x}:=\mathbf{C}[\{x\}]=\{y \mid(x, y) \in \mathbf{C}\}$ zuordnet. Der Operator $\mathbf{C}$ soll eineindeutig sein und Urelementen eigentliche Klassen zuordnen:

R5: $\quad \mathbb{E} \mid x, y \wedge \mathbf{C} x=\mathbf{C} y \Rightarrow x=y$,

R6: $\quad \operatorname{Ur} x \Rightarrow \operatorname{Pcl} C$,

R7: $\quad \operatorname{Mg} x \Rightarrow x=\mathbf{C} x$.

R7 ist technischer Natur und wird durch eine Definition erweitert:

$$
\operatorname{Pcl} x \Rightarrow \mathbf{C} x:=x \text {. }
$$

Dann gilt also

$$
\mathrm{Cl} \mathrm{C}_{\mathrm{C}}
$$


Die „Bilder” der Elemente unter C nennen wir C-Klassen:

$$
\begin{aligned}
\mathrm{C}-\mathrm{Cl} x & : \Leftrightarrow \bigvee_{\mathrm{El} y} x=\mathbf{C} y, \\
\mathrm{C}-\mathrm{Pcl} x & \Leftrightarrow \bigvee_{\mathrm{Ur} y} x=\mathbf{C} y .
\end{aligned}
$$

Nach R5 läßt sich die Zuordnung $\mathbf{C}$ auf den $\mathbf{C}$-Klassen umkehren. Die Umkerzuordnung bezeichnen wir mit e (Elementfunktion)

$$
\text { C. } \mathrm{Cl} x \Rightarrow \mathrm{Ele} \wedge \wedge \mathrm{Ce} x=x \text {. }
$$

Aus technischen Erwägungen definieren wir e auch noch für Urelemente

$$
\text { Ur } x \Rightarrow \mathbf{e} x:=x \text {. }
$$

Dann gilt:

$$
\text { C. } \mathrm{Cl} x \vee \mathrm{Ur} x \Rightarrow \mathrm{Elex} .
$$

Die Wirkungsweise von $\mathbf{C}$ und $\mathbf{e}$ läßt sich im folgenden Diagramm veranschaulichen:

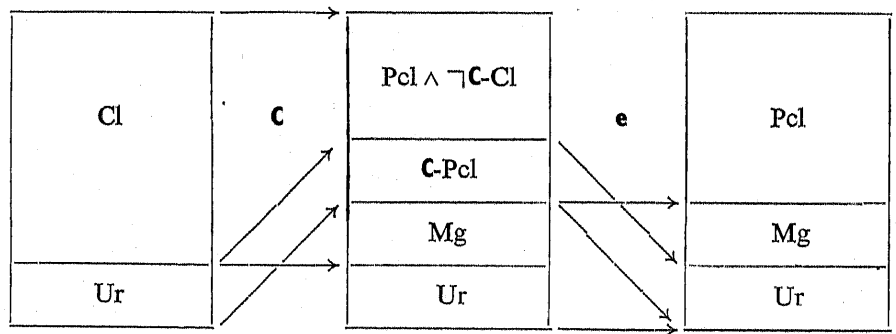

Aus den bisherigen Axiomen läßt sich die Existenz von Urelementen und eigentlichen C-Klassen noch nicht beweisen, denn die reine NBG-Theorie mit $\{(x, y) \mid y \in x\}$ als „C" ist ein Modell der bisherigen Axiome. Bevor wir jedoch die Existenzgewisser eigentlicher C-Klassen fordern, soll die Grundidee dieser Arbeit, eigentliche C-Klassen als Elemente zu interpretieren, präzisiert werden, woraus sich dann in natürlicher Weise eine Reihe von Axiomen für die Bildung von C-Klassen ergeben.

2.1. Die modulierte Theorie. Im Uberblick haben wir bereits gesagt, wie wir eine C-Klasse $x$ wieder als Element auffassen wollen: wir interpretieren ,ex $\in y^{\prime}$ als „x ist Element von $y$ ". Dies läuft gerade darauf hinaus, da $\beta$ wir die C-Klasse $x$ mit dem zugehörigen Element ex identifizieren und dann die Elementrelation modulo dieser Identifizierung betrachten. Die Identifikation wird durch die folgende Äquivalenzrelation geliefert:

$$
x \stackrel{c}{=} y: \Leftrightarrow C_{x}=C y .
$$

Wir betrachten jetzt alle Grundbegriffe der NBG-Theorie modulo der Äquivalenzrelation $\stackrel{c}{=} "$ (lies: $c$-gleich) indem wir definieren:

$$
\begin{aligned}
x \in_{c} y & \Leftrightarrow \bigvee_{u} \bigvee_{v}(x \stackrel{c}{=} u \wedge y \stackrel{c}{=} v \wedge u \in v), \\
\mathrm{El}_{c} x & \Leftrightarrow \bigvee_{u}(x \stackrel{c}{=} u \wedge \mathrm{El} u) \\
\mathrm{Cl}_{c} x & \Leftrightarrow \bigvee_{u}(x \stackrel{c}{=} u \wedge \mathrm{Cl} u) .
\end{aligned}
$$

Damit ist eine "Interpretation" der NBG-Grundbegriffe in der C-Theorie gegeben und es liegt nahe zu fragen, welche NBG-Axiome bei dieser „Interpretation” gelten. Hierzu definieren wir (analog der Relativierung bei inneren Modellen) rekursiv über den Aufbau zu jeder NBG-Formel $\mathfrak{2}$ ihre Modulation $\mathfrak{H}_{c}$, die C-Formel ist:

$$
\begin{aligned}
(x \in y)_{c} & : \equiv x \in_{c} y, \\
(\mathrm{El} x)_{c} & : \equiv \mathrm{El}_{c} x, \\
(\mathrm{Cl} x)_{c} & : \equiv \mathrm{Cl}_{c} x, \\
(\neg A)_{c} & : \equiv \neg A_{c}, \\
(A \wedge B)_{c} & : \equiv A_{c} \wedge B_{c}, \\
(\bigwedge A)_{c} & : \equiv \bigwedge_{x} A_{c} .
\end{aligned}
$$

Zunächst stellen wir fest, daß die Modulationen der Rahmenaxiome gelten:

$$
\begin{aligned}
x \in y & \Rightarrow x \in_{c} y, \\
\mathrm{El} x & \Rightarrow \mathrm{El}_{c} x, \\
\mathrm{Cl} x & \Rightarrow \mathrm{Cl}_{c} x, \\
x \in_{c} y & \Rightarrow \mathrm{El}_{c} x \wedge \mathrm{Cl}_{c} y, \\
\mathrm{El}_{c} x & \Rightarrow \bigvee_{y} x \in_{c} y, \\
\mathrm{El}_{c} x & \vee \mathrm{Cl}_{c} x .
\end{aligned}
$$

Bevor wir die „modulierte” Theorie weiteruntersuchen, stellen wir einige elementare Eigenschaften zusammen, die im folgenden laufend benötigt werden. 
Somit ist e für alle $x$ mit $\mathbf{E l}_{c} x$ definiert!

$$
\begin{gathered}
x \in_{c} y \Leftrightarrow \mathbf{e} x \in \mathbf{C} y, \\
\mathrm{Cl}_{c} x, \\
x \in_{c} y \Leftrightarrow \bigvee_{u}(x \stackrel{c}{=} u \wedge u \in \mathbf{C} y), \\
\mathrm{El} x \Rightarrow\left(x \in_{c} y \Leftrightarrow x \in \mathbf{C} y\right), \\
\mathrm{El} x \wedge \mathrm{Cl} y \Rightarrow\left(x \in_{c} y \Leftrightarrow x \in y\right) .
\end{gathered}
$$

Die Eigenschaften sind per Definition trivial oder folgen aus den jeweils vorangegangenen. Wir zeigen jetzt, daß (erwartungsgemäß) die modulierte Gleichheit gerade die Äquivalenzrelation „„ $=$ " ist, und daß das modulierte Extensionalitätsaxiom gilt:

$$
\begin{gathered}
(x=y)_{c} \Leftrightarrow x \stackrel{c}{=} y, \\
\bigwedge_{z}\left(z \in_{c} x \Leftrightarrow z \in_{c} y\right) \Rightarrow x \stackrel{c}{=} y .
\end{gathered}
$$

$\left(\mathbf{E X T}_{c}\right)$

Beweis (2.1.21): Man betrachte nur Elemente $z$ und wende (2.1.18) an

Beweis (2.1.20): „ $\Rightarrow$ mit (2.1.21), „«" ist klar!

Speziell folgt, daß $c$-gleiche Objecte in modulierten C-Formeln $\mathfrak{A}_{c}$ äquivalent durcheinander ersetzbar sind. Hieraus erhält man für $\mathbf{C}-$ Formeln $\mathfrak{A}$ :

$$
\begin{aligned}
& \bigwedge_{\mathrm{Cl}_{o} x} \mathfrak{P}_{c} \Leftrightarrow \bigwedge_{\mathrm{Cl} x} \mathfrak{R}_{c}, \\
& \bigwedge_{\mathbb{E l}_{c} x} \mathfrak{I}_{c} \Leftrightarrow \bigwedge_{\mathbb{E} 1 x} \mathfrak{I}_{c}, \\
& \bigwedge_{\mathrm{El}_{c} x} \mathfrak{T}_{c} \Leftrightarrow \bigwedge_{-\mathrm{Cl} x} \mathfrak{P T}_{c} .
\end{aligned}
$$

Die Beweise von (2.1.22) -(2.1.23) sind klar. Bei (2.1.24) beachte man (2.1.14) und daß Urelemente zu C-Klassen $c$-gleich sind.

Es soll jetzt gezeigt werden, daß auch das modulierte Klassenkomprehensionsaxiomschema gültig ist:

(2.1.25) Für eine C-Formel $\mathfrak{2}(x)$ im engeren. Sinn gilt:

$$
\mathrm{Cl}_{c}\left\{x \mid \mathfrak{N}_{c}(x)\right\}, \quad \bigwedge_{\mathbb{E l}_{o} z}\left(z \in_{c}\left\{x \mid \mathfrak{Q N}_{c}(x)\right\} \Leftrightarrow \mathfrak{Q}_{c}(z)\right) .
$$

Beweis (2.1.25): Man beachte, daß die Quantoren in $\mathfrak{H}_{c}(x)$ genau dann auf $\mathrm{EI}_{c}$ relativiert sind, wenn $\mathfrak{Q}(x)$ Formel im engeren Sinn ist.

Man kann nụn wieder jede Definition der NBG-Theorie, die nur auf der Gültigkeit der Axiome R1-R3, EXT, KOMP basiert, durch die „Interpretation” bzw. "Modulation” (2.2.1)-(2.2.4) bzg1. „ $\stackrel{\circ}{=}$ modulieren (analog dem relativieren bei inneren Modellen). Die Modulation eines Begriffes wird durch den Index , $c$ " bezeichnet. Aus (2.1.25) erhält man z. B. die modulierte Klassenkomprehension:

$$
\left\{x \mid \mathfrak{A}_{c}(x)\right\}_{c} \stackrel{c}{=}\left\{x \mid \mathfrak{A}_{c}(x)\right\} .
$$

Begriffe, die in der modulierten Theorie mit ihrer Modulation übereinstimmen, heißen absolut (bzgl $\stackrel{c}{=}$ ). So ist $\mathrm{z}$. B. die Klassenkomprehension absolut, aber die Gleichheit und die Begriffe $\mathrm{El}, \mathrm{Mg}, \mathrm{Cl}$ nicht.

Es taucht jetzt die Frage auf, ob auch die Modulationen der Mengenbildungsaxiome gültig sind. Hierzu zeigen wir zunächst:

$$
\begin{gathered}
\varnothing_{c} \stackrel{c}{=} \varnothing, \\
\mathrm{El}_{c} x, y \Rightarrow\{x, y\}_{c} \stackrel{c}{=}\{\mathbf{e} x, \mathbf{e} y\}, \\
U_{c} x \stackrel{c}{=} \bigcup_{y \in \mathrm{C} c} \mathbf{C}_{y}, \\
x \subset_{c} y \Leftrightarrow \mathrm{C} x \subset \mathbf{C} y, \\
P_{c} x \stackrel{c}{=}\{y \mid \mathbf{C} y \subset \mathbf{C} x\} .
\end{gathered}
$$

Auf die einfachen Beweise unter Verwendung von (2.1.26) und (2.1.5)-(2.1.14) können wir verzichten.

Hieraus erhält man die Gültigkeit der Modulationen vom Axiom der leeren Menge und Zweiermengenaxiom:

$$
\begin{gathered}
\mathrm{Mg}_{c} \varnothing_{c}, \\
\mathrm{El}_{c} x, y \Rightarrow \operatorname{Mg}_{c}\{x, y\}_{c} .
\end{gathered}
$$

Unter Berücksichtigung von (vgl. (2.1.16))

$$
\mathrm{Mg}_{c} x \Leftrightarrow \mathrm{El}_{c} x
$$

erhält man mit (2.1.24) die folgenden Kriterien für die Gültigkeit der Modulationen von Vereinigungs-, Potenzmengen- und Aussonderungsaxiom:

$$
\begin{aligned}
& \mathbf{V M}_{c} \Leftrightarrow \bigwedge_{\mathrm{C}-\mathrm{Cl} x} \mathbf{C}-\mathrm{Cl} \bigcup_{y \in x} \mathbf{C} y, \\
& \mathbf{P M}_{c} \Leftrightarrow \bigwedge_{\mathrm{C}-\mathrm{Cl} x} \mathbf{C}-\mathrm{Cl}\{y \mid \mathbf{C} y \subset x\}, \\
& \mathbf{A U S}_{c} \Leftrightarrow \bigwedge_{\mathrm{C}-\mathrm{Cl} x} \bigwedge_{\mathrm{Cl} y}(y \subset x \Rightarrow \mathrm{C}-\mathrm{Cl} y) .
\end{aligned}
$$


Abschließend untersuchen wir noch die Modulationen von Unendlichkeit- und Ersetzungsaxiom, es gilt:

$$
\begin{gathered}
\omega_{c} \stackrel{c}{=} \omega, \\
\operatorname{Mg}_{c} \omega_{c}, \\
\mathbf{E R S}_{c} \Leftrightarrow \bigwedge_{\mathrm{C}-\mathrm{Clx}} \bigwedge_{\mathrm{Cl} y} \bigwedge_{f: x \rightarrow y} \mathbf{C}-\mathrm{Cl} f[x] .
\end{gathered}
$$

Die Beweise ergeben sich aus den entsprechenden Definitionen. Bisher haben wir gesehen, daß die Modulation bzgl. $\stackrel{c}{=}$ als Präzisierung der Idee, Klassen und Elemente zu identifizieren, wieder "fast” eine NBG-Theorie ist. Die fehlenden Eigenschaften $\mathbf{V M}_{c}, \mathbf{P M}_{c}, \mathbf{A U S}_{c}$ werden wir daher als Axiome für C-Klassen im nächsten Abschnitt fordern (warum ERS $c$ ausgelassen wird, zeigt sich später).

2.2. Die Axiome der C-Klassen-Bildung. In Anlehnung an die Betrachtungen der modulierten C-Theorie (insbesondere (2.1.34)-(2.1.36)) formulieren wir jetzt einige Axiome zur Bildung von C-Klassen:

Vereinigungsaxiom für C-Klassen.

VC: $\quad \mathbf{C}-\mathrm{Cl} x \Rightarrow \mathrm{C}-\mathrm{Cl} \underset{x \in y}{\cup} \mathbf{C}$.

Potenzaxiom für C-Klassen

PC: $\mathbf{C}-\mathrm{Cl} x \Rightarrow \mathbf{C}-\mathbf{C l}\{y \mid \mathbf{C} y \subset x\}$.

Aussonderungsaxiom für $\mathbf{C}-$ Klassen

AUSC: C-Cl $x \wedge \mathrm{Cl} y \wedge y \subset x \Rightarrow \mathrm{C}-\mathrm{Cl} y$.

Wir formulieren auch gleich noch das Ersetzungsprinzip für C-Klassen, fordern es aber nicht als Axiom:

\section{Ersetzungsprinzip für C-Klassen}

ERSC: $\quad \mathbf{C}-\mathrm{Cl} x \wedge \mathrm{Cl} y \wedge f: x \rightarrow y \mathrm{Abb} \Rightarrow \mathbf{C}-\mathrm{Cl} f[x]$.

Analog der Russell'schen Antinomie erhält man aus AUSC sofort die Existenz von Klassen, die keine C-Klassen sind.

$$
\text { ᄀC-ClE, }
$$

Beweise: $R:=\{\{x\} \mid\{x\} \notin C x\} \subset \underline{V} \subset \underline{E}$ ist keine C-Klasse, denn sonst würde gelten: $\{\mathbf{e} R\} \in R \Leftrightarrow\{\mathbf{e} R\} \notin R$.

Für eine Klasse $L$ können wir Relationen $K \subset L \times \underline{E}$ als „Familien

$$
(K(z):=K[\{z\}])_{z \in L}
$$

von Klassen" auffassen.
Mit dieser Terminologie ergibt sich:

(2.2.2) Für eine Menge $x$ und eine Familie $\left(K_{y}\right)_{y \in x}$ von Klassen gilt:

$$
\bigwedge_{y \in x} \mathrm{C}-\mathrm{Cl} K_{y} \Rightarrow \mathrm{C} \cdot \mathrm{Cl} \bigcup_{y \in x} K_{y} .
$$

Beweis (2.2.2): Nach dem Ersetzungsaxiom ist $\left\{\mathbf{e} K_{y} \mid y \in x\right\}$ eine Menge, also C-Klasse, und es folgt, daß $\bigcup_{y \in x} \mathrm{Ce} K_{y}=\bigcup_{y \in x} K_{y}$ eine C-Klasse ist.

Aber auch durch die neuen C-Klassen-Bildungsaxiome ist die Existenz von eigentlichen C-Klassen (bzw. Urobjekten) noch nicht gesichert. Es bleibt also zu überlegen, von welchen Klassen man fordern sollte, daß sie C-Klassen sind. Im Hinblick auf die Verwendbarkeit der C-Theorie als mengentheoretische Grundlage der Kategorientheorie sollte zumindest die Klasse $\mathrm{V}(\varnothing)=\mathrm{E}(\varnothing)$ der Mengen, die ohne Urelemente aufgebaut sind (also die Klassen aller Mengen in einer reinen NBG-Theorie) eine C-Klasse sein. Aber das wird noch nicht ausreichen, sondern wir wollen, daß mit jeder Menge $x$ von Urelementen (bzw. von C-Klassen in der modulierten Theorie) auch die Klasse $\mathrm{E}(x)$ aller mit Hilfe von $x$ aufgebauten Mengen eine C-Klasse ist. Schließlich kann man noch einen Schritt weitergehen und fordern, daß für jede C-Klasse $U \subset \underline{U}$ die Klasse $\underline{E}(U)$ noch eine C-Klasse ist. Wir geben eine dazu äquilvalente Bedingung an, die wir dann als Axiom formulieren:

$$
\bigwedge_{U \in \underline{U}}(\mathrm{C}-\mathrm{Cl} U \Rightarrow \mathrm{C}-\mathrm{ClE}(U)) \Leftrightarrow \bigwedge_{\mathrm{Cl} x}(\mathrm{C}-\mathrm{ClFd}(x) \Rightarrow \mathrm{C}-\mathrm{Cl} x) .
$$

Beweis (2.2.3):

„": C-ClFd $(x) \wedge x \subset \underline{\mathrm{E}}(\mathrm{Fd}(x)) \Rightarrow \mathrm{C}-\mathrm{Cl} x$.

"Е: $\mathrm{Fd} \underline{\mathrm{E}}(U)=U$.

Damit kommen wir zum letzten Axiom für die Bildung von C-Klassen:

\section{Fundamentaxiom für C-Klassen}

FD: $\quad \mathrm{Cl} x \wedge \mathrm{C}-\mathrm{ClFd}(x) \Rightarrow \mathrm{C}-\mathrm{Cl} x$.

Analog $(1.1 .1)-(1.1 .5)$ erhält man die leichte Verschärfung:

$$
\mathrm{Cl} x \Rightarrow(\mathbf{C}-\mathrm{Cl} x \Leftrightarrow \mathrm{C}-\mathrm{ClFd}(x)) .
$$

Weil $\underline{E}=\underline{E}(\underline{U})$ keine $C$-Klasse ist, kann jetzt auch $\underline{U}$ keine C-Klasse mehr und erst recht keine Menge mehr sein:

$$
\neg \mathrm{C}-\mathrm{Cl} \underline{\mathrm{U}} .
$$

Wir wollen jetzt die Stärke des Fundamentaxioms etwas näher kennenlernen. $\mathrm{Zu}$ jeder C-Klasse $A$ erhält man mit $\mathbf{F D}$ eine wesentlich größere $\mathbf{C}$-Klasse

$$
\underline{\mathrm{E}}(\{\mathbf{e x} \mid \operatorname{Pc} 1 x \wedge x \in A\}) \text {. }
$$


Durch Iteration erhält man eine Folge $A_{0}, A_{1}, \ldots$ von C-Klassen mit:

$$
A_{0}:=\underline{\mathrm{E}}(\varnothing), \quad A_{n+1}:=\underline{\mathrm{E}}\left(\underline{\mathrm{U}} \cap P_{c} A_{n}\right)
$$

wobei allgemein $P_{c} K:=\{y \mid \mathbf{C} y \subset \mathrm{C} K\}$ für C-Klassen $K$, vgl. (2.1.30).

Nach (2.2.2) ist aber auch $A_{\omega}:=\bigcup_{n \in \omega} A_{n}$ eine C-Klasse, und das Verfahren läßt sich weiter fortsetzen. Wir präzisieren dies in der folgenden Definition:

(2.2.6) Die Familie $\left(\underline{A}_{a}\right)_{a \in \Omega}$ von $\mathbf{C}-$ Klassen wird rekursiv definiert:

$$
\bigwedge_{a \in \Omega} \underline{\mathrm{A}}_{a}:=\underline{\mathrm{E}}\left(\underline{\mathrm{U}} \cap \bigcup_{b<a} P_{c} \underline{\mathrm{A}}_{b}\right) .
$$

Zur Vereinfachung definieren wir:

$$
\bigwedge_{a \in \Omega} \underline{\mathrm{B}}_{a}:=\underline{\mathrm{U}} \cap \underline{\mathrm{A}}_{a}=\underline{\mathrm{U}} \cap \bigcup_{b<a} P_{c} \underline{\mathrm{A}}_{b} .
$$

Dann gilt für $a \in \Omega$ :

(2.2.8) $\underline{\mathrm{A}}_{a}$ ist normales inneres Modell mit $\underline{\mathrm{U}}_{\mathbf{A}_{a}}=\underline{\mathbf{B}}_{a}$.

Für $a, b \in \Omega$ notieren wir noch einige Folgerungen:

$$
\begin{gathered}
a \leqslant b \Rightarrow \underline{\mathrm{A}}_{a} \subset \underline{\mathrm{A}}_{b} \wedge \underline{\mathbf{B}}_{a} \subset \underline{\mathrm{B}}_{b}, \\
P \underline{\mathrm{A}}_{a}=\underline{\mathrm{A}}_{a} \backslash \underline{\mathrm{B}}_{a}, \\
\underline{\mathrm{B}}_{0}=\varnothing, \\
a \text { Limeszahl } \Rightarrow \underline{\mathrm{B}}_{a}=\underline{\cup}_{b<a} \underline{\mathrm{B}}_{b}, \\
\mathbf{C}-\mathrm{Cl} \underline{\mathrm{A}}_{a}, \underline{\mathrm{B}}_{a} \\
\underline{\mathrm{U}} \cap P_{c} \underline{\mathrm{A}}_{a}=\underline{\mathrm{B}}_{a+1}, \\
P_{c} \underline{\mathrm{A}}_{a}=\underline{\mathrm{B}}_{a+1} \cup \underline{P}_{a} \subset \underline{\mathrm{A}}_{a+1}, \\
U_{c} \underline{\mathrm{A}}_{a} \subset \underline{\mathrm{A}}_{a} .
\end{gathered}
$$

Beweise (2.2.9)-(2.2.16): (2.2.9)-(2.2.12) ist klar, (2.2.13) folgt durch Induktion, (2.2.14) ist klar, (2.2.15) folgt aus (2.2.14), und für (2.2.16) zeigen wir, daß für $x \in \underline{A}_{a}$ gilt: $\mathbf{C} x \subset \mathrm{A}_{a}$. Falls $x$ Menge ist, folgt mit (2.2.10) $\mathbf{C} x=x \subset \mathrm{A}_{a}$. Falls $x$ Urelement ist, so ist $x \in \mathrm{B}_{a}$, und es gibt ein $b<a$ mit $x \in P_{c} \mathrm{~A}_{b}$, also $\mathrm{C} x \subset \underline{\mathrm{A}}_{b} \subset \mathrm{A}_{a}$.

Die sukzessive Konstruktiou der $\mathrm{A}_{a}$ und $\mathrm{B}_{a}$ läßt sich im folgenden Diagramm veranschaulichen:

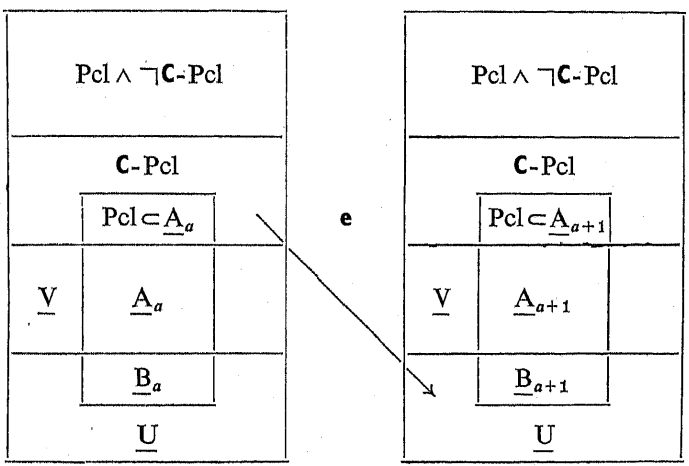

Jetzt haben wir mit $\left(\mathbf{A}_{a}\right)_{a \in \Omega}$ eine relativ "große" Familie von $\mathbf{C}$-Klassen gefunden Es taucht daher die Frage auf, ob es außer den Teilklassen der $\underline{A}_{a}$ 's überhaupt noch weitere C-Klassen gibt. So braucht z.B. $\bigcup_{a \in \Omega} \underline{A}_{a}$ keine C-Klasse mehr zu sein, außer wenn wir das Ersetzungsprinzip ERSC voraussetzen. Im nächsten Abschnitt werden wir zeigen, da $\beta$ die Existenz von C-Klassen, die keine Teilklassen der $\underline{\mathrm{A}}_{a}$ 's sind, nicht aus den bisherigen Axiomen folgt.

2.3. Das Abschlußprinzip. Jetzt formulieren wir das Abschlußprinzip (ohne es als Axiom zu fordern), welches besagt, da $B$ jede $C$-Klasse bereits Teilklasse einer C-Klasse der Form $\underline{A}_{a}$ ist:

\section{Abschlussprinzip}

AP $\quad \mathrm{C}-\mathrm{Cl} x \Rightarrow \bigvee_{a \in \Omega} x \subset \mathrm{A}_{a}$.

Wir werden zeigen, daß AP relativ widerspruchsfrei zu den bisherigen Axiomen ist und dann einige Konsequenzen von $\mathbf{A P}$ andeuțen. Zunächst definieren wir:

$$
\begin{gathered}
\underline{\mathrm{A}}(\Omega):=\bigcup_{a \in \Omega} \underline{\mathrm{A}}_{a}, \\
\underline{\mathrm{B}}(\Omega):=\underline{\mathrm{A}}(\Omega) \cap \underline{\mathrm{U}}=\bigcup_{a \in \Omega} \underline{\mathrm{B}}_{a} .
\end{gathered}
$$

A $(\Omega)$ is normales inneres Modell mit $\underline{\mathrm{B}}(\Omega)$ als Klasse der Urelemente.

$$
\underline{\mathrm{E}}(\mathrm{B}(\Omega))=\underline{\mathrm{A}}(\Omega) \text {. }
$$

Beweis (2.3.2): Für Urelemente $x$ gilt:

$$
x \in \underline{\mathrm{E}}(\mathrm{B}(\Omega)) \Leftrightarrow x \in \underline{\mathrm{B}}(\Omega) \Leftrightarrow x \in \underline{\mathrm{A}}(\Omega) .
$$

Für Mengen $x$ gilt.

$$
x \in \underline{\mathrm{E}}(\mathrm{B}(\Omega)) \Leftrightarrow \mathrm{Fd} x \subset \underline{\mathrm{B}}(\Omega) \Leftrightarrow \bigvee_{a \in \Omega} \mathrm{Fd} x \subset \underline{\mathrm{B}} a \underline{\bigvee}_{a} x \in \underline{\mathrm{E}}\left(\underline{\mathrm{B}}_{a}\right)=\underline{\mathrm{A}}_{a} \Leftrightarrow x \in \underline{\mathrm{A}}(\Omega) .
$$

2- Fundamenta Mathematicae, T. XCrr 
Damit haben wir zwei äquivalente Formulierungen des Abschlußprinzips gewonnen.

$$
\mathbf{A P} \Leftrightarrow \underline{\mathrm{E}}=\underline{\mathrm{A}}(\Omega) \Leftrightarrow \underline{\mathrm{U}}=\underline{\mathrm{B}}(\Omega) .
$$

Beweis (2.3.3): Die zweite Äquivalenz folgt aus (2.3.1)-(2.3.2) und zur ersten zeigen wir allgemeiner:

$$
x \in \underline{\mathrm{A}}(\Omega) \Leftrightarrow \bigvee_{a \in \Omega} \mathrm{C} x \subset \underline{\mathrm{A}}_{a} .
$$

Beweis (2.3.4):

" $\Rightarrow$ : Es gibt ein $a \in \Omega$ mit $x \in \underline{\mathrm{A}}_{a}$, also $\mathrm{C} x \subset \cup_{c} \underline{\mathrm{A}}_{a} \subset \underline{\mathrm{A}}_{a}$ (vgl. (2.2.6)).

„«": Es ist $x \in P_{c} \underline{\mathrm{A}}_{a} \subset \underline{\mathrm{A}}_{a+1} \subset \mathrm{A}(\Omega)$ (vgl. (2.2.5))

Die relative Widerspruchsfreiheit von $\mathbf{A P}$ läßt sich jetzt wegen (2.3.3) leicht unter Verwendung der Standardmethode der inneren Modelle zeigen. Zunächst ist $\mathrm{A}(\Omega)$ nach (2.3.2) ein normales inneres Modell der NBG-Theorie. Wir interpretieren auch noch $\mathbf{C}$ über $\underline{\mathrm{A}}(\Omega)$ und zeigen, $\operatorname{da} \beta \underline{\mathrm{A}}(\Omega)$ dann Modell der C-Theorie mit Abschlußprinzip ist. Hierbei gehen wir etwas allgemeiner vor, indem wir $\mathbf{C}$ auf eine beliebige Klasse $K$ relativieren.

$$
\mathrm{C}_{K}:=\mathbf{C} \cap(K \times K) .
$$

Nach (1.2.4) und (2.3.5) können wir jetzt auch jeder C-Formel $\mathfrak{A}$ ihre Relativierung $\mathfrak{A}_{\mathrm{K}}$ zuordnen.

Falls $K$ abgeschlossen bzgl. C ist (d.h. für $x \in E$ ist $\mathbf{C} x \subset K$ ), so gelțen die Relativierung der Rahmenaxiome R4-R7:

(2.3.6) $K$ heißt C-abgeschlossen: $\Leftrightarrow \bigwedge_{x \in K} \mathbf{C} x \subset K$,

(2.3.7) $K$ C-abgeschlossen $\Rightarrow(\mathbf{R} 4 \wedge \mathbf{R 5} \wedge \mathbf{R 6} \wedge \mathbf{R 7})_{K}$.

Zum Beweis von (2.3.7) genügt es, festzustellen, daß $\mathbf{C}$ absolut ist:

(2.3.8) $K$ C-abgeschlossen $\Rightarrow \bigwedge_{x \in \mathrm{K}} \mathbf{C}_{K} x=\mathbf{C}_{x}$.

Die Relativierungen der C-Klasserbildungsaxiome gelten nicht für jedes $\mathbf{C}$-abgeschlossene $K$. Falls $K$ normales inneres Modell ist, so hat man aber folgende Kriterien:

(2.3.9) - Ist $K \mathrm{C}$-abgeschlossenes, normales inneres Modell, so gilt

(a) $\quad \mathbf{V C}_{K} \Leftrightarrow \bigwedge_{\mathrm{C}-\mathrm{Cl}_{K} x} \mathrm{C}_{-} \mathrm{Cl}_{K} \bigcup_{y \in x} \cup \mathbf{C}_{y}$,

(b) $\quad \mathbf{P C}_{K} \Leftrightarrow \widehat{\mathrm{C}} \mathrm{C}_{\mathrm{Cl}} \mathrm{Cl}_{K}\{y \mid \mathbf{C} y \subset x\}$,

(c) $\quad \operatorname{AUSC}_{K} \Leftrightarrow \bigwedge_{\mathrm{C}-\mathrm{Cl} x} x \bigwedge_{\mathrm{Cl} y}\left(y \subset x \Rightarrow \mathrm{C}-\mathrm{Cl}_{K} y\right)$,

(d) $\quad \mathbf{F D}_{K} \Leftrightarrow \bigwedge_{\mathbf{C l}_{\mathbf{K}} \boldsymbol{x}}\left(\mathbf{C}-\mathrm{Cl}_{K} \mathrm{Fd}(x) \Rightarrow \mathbf{C}-\mathrm{Cl}_{K} x\right)$.
Beweis (2.3.9): Folgt aus (2.3.8) und der Absolutheit von Elementrelation, Vereinigung, Inklusion und Fundament für normale innere Modelle.

Als Ergänzung zu (2.3.9) stellen wir noch fest

(2.3.10) $K$ C-abgeschlọssen $\Rightarrow\left(C_{-}-\mathrm{Cl}_{K} x \Leftrightarrow \mathrm{C}-\mathrm{Cl} x \wedge \mathbf{e} x \in K\right)$.

Diese Ergebnisse wenden wir jetzt auf $K=\underline{\mathrm{A}}(\Omega)$ an und erhalten:

(2.3.11) $\mathrm{A}(\Omega)$ ist $\mathbf{C}$-abgeschlossenes, normales inneres Modell,

$$
\text { C. }-\mathrm{Cl}_{\mathbf{A}(\Omega)} x \Leftrightarrow \bigvee_{a \in \Omega} x \subset \underline{\mathrm{A}}_{a},
$$

$$
(\mathrm{VC} \wedge \mathrm{PC} \wedge \mathrm{AUSC} \wedge \mathrm{FD})_{\underline{A}(\Omega)} .
$$

Beweis (2.3.10)-(2.3.11): vgl. (2.3.4) und (2.3.10).

Beweis (2.3.13): Anwendung von (2.3.9) und (2.3.12). $\mathrm{Zu} \mathrm{VC}$ und $\mathbf{P C}$ betrachte man (2.2.15) und (2.2.16), AUS ist klar, und FD erkennt man wie folgt: Ist $\mathrm{Fd}(x) \subset \mathrm{A}_{a}$ für eine Klasse $x$, so folgt $\mathrm{Fd}(x) \subset \mathrm{B}_{a}$, also $x \subset E\left(\underline{\mathrm{B}}_{a}\right)=\mathrm{A}_{a}$ (mit 1.1.8). Damit ist $\mathrm{A}(\Omega)$ in natürlicher Weise ein Modell der C-Theorie. Es bleibt noch zu zeigen, da $\bar{\beta}$ die Relativierung des Abschlußprinzips gilt:

$$
\mathbf{A P}_{\underline{A}(\Omega)} \text {. }
$$

Beweis (2.3.14): Man zeigt, daß $\underline{A}$ absolut ist, d.h. $\underline{A}=\underline{A}_{\mathbf{A}(\Omega)}$, indem man schrittweise die Absolutheit der zur Definition von A benötigten Begriffe beweist.

Nachdem nun die relative Widerspruchsfreiheit von AP zu den Axiomen gezeigt, bleibt es uns überlassen, ob wir es als Axiom fordern wollen oder nicht. Der Haupteinwand gegen AP als Axiom ist, da $\mathrm{B}$ es dem Ersetzungsprinzip für C-Klassen widerspricht.

$$
\text { (2.3.15) }
$$

$$
\text { ERSC } \Rightarrow \neg \text { AP. }
$$

Beweis (2.3.15): Nach ERSC wäre $\underline{\mathrm{A}}(\Omega)$ eine C-Klasse, also $\underline{\mathrm{A}}(\Omega) \neq \underline{\mathrm{E}}(2.2 .1)$ und somit $\neg \mathbf{A P}$ (2.3.3)

Ob man AP oder ERSC als zusätzliches Axiom fordern soll hängt davon ab, was man dadurch erreichen will. Für eine zufriedenstellende Entwicklung der Kategorientheorie innerhalb der C-Theorie zum Beispiel, sind weder AP noch ERSC unbedingt erforderlich (vgl. § 4) und wir verzichten daher auf sie als Axiome.

Abschießend geben wir noch eine äquivalente Formulierung des Abschlußprinzips an, die modelltheoretischen Charakter hat und besagt, daß es keine nichttrivialen C-abgeschlossenen Modelle der Form $\underline{\mathrm{E}}(U)$ mit $U \subset \underline{\mathrm{U}}$ gibt.

(2.3.15) Die folgenden beiden Formeln sind äquivalent

(a) AP.

(b) Für jedes $U \subset \underline{U}$ gilt:

$$
\underline{E}(U) \text { C-abgeschlossen } \wedge(\mathbf{V C} \wedge \mathbf{P C} \wedge \mathbf{A U S C} \wedge \mathbf{F D})_{\mathbb{B}(U)} \Rightarrow U=\underline{U} .
$$


Beweis (2.3.15):

(a) $\Rightarrow$ (b). Durch Induktion zeigt man $\underline{\mathrm{B}}_{a} \subset U$ für $a \in \Omega$, und erhält mit 2.3.3 $\underline{\mathrm{U}}=\underline{\mathrm{B}}(\Omega) \subset U$.

$(\overrightarrow{\mathrm{a}}) \Leftarrow(\mathrm{b})$ : Für $U=\underline{\mathrm{B}}(\Omega)$ folgt wegen $(2.3 .11)$, (2.3.13) sofort $\underline{\mathrm{U}}=\underline{\mathrm{B}}(\Omega)$ und somit AP nach 2.3.3.

2.4. Die weitere Entwicklung der C-Theorie. Es soll jetzt in einigen Stichpunkten auf die weitere Entwicklung der C-Theoric eingegangen werden. Hierbei geht es in erster Linie darum, die für Mengen bekannten Begriffe und Eigenschaften auf C-Klassen auszudehnen. Dies geschieht unter Verwendung der modulierten Theorie und ganz grob kann man sagen, daß alle Begriffe und Eigenschaften der Zermelo' schen Mengenlehre sich auf $\mathbf{C}$-Klassen über tragen lassen. Um die Leistungsfähigkeit der modulierten Theorie $\mathrm{zu}$ verdeutlichen, geben wir einige exemplarische Beispiele.

Zunächst stellen wir fest, daß kartesische Produkte von C-Klassen wieder C-Klassen sind und können dann die Klasse aller Abbildungen zwischen zwei C-Klassen definieren.

$$
\begin{aligned}
& \mathrm{C}-\mathrm{Cl} K, L \Rightarrow \mathrm{C}-\mathrm{Cl} K \times L, \\
& \mathrm{C}-\mathrm{Cl} K, L \Rightarrow \mathrm{Abb}_{c}(K, L):=\{\mathbf{e} f \mid f: K \rightarrow L \mathrm{Abb}\} .
\end{aligned}
$$

Man beachte, daß man für beliebige Klassen $K, L$ die Klasse der Abbildungen $K \rightarrow L$ nicht unbedingt bilden kann. Es soll jetzt gezeigt werden, daß man für C-Klassen auch ein geordnetes Par definieren kann, welches ein Element ist, so daß man dann auch Abbildungen mit C-Klassen als Argumente und Werte hat (in [3] sind geordnete Paare von Klassen wieder Klassen).

$$
\begin{aligned}
& \text { C-Cl } x, y \Rightarrow(x, y)_{c}:=(\mathbf{e} x, \mathbf{e} y), \\
& \text { C-Cl } x, y, u, v \wedge(x, y)_{c}=(u, v)_{c} \Rightarrow x=u, y=v .
\end{aligned}
$$

Eine Abbildung $f: K \rightarrow L$ kann nun auch interpretiert werden als eine Abbildung zwischen den $\mathbf{C}$-Klassen $\mathbf{C} x$ mit $x \in K$ und den $\mathbf{C}$-Klassen $C_{y}$ mit $y \in L$.

Neben diesen elementaren Begriffen wollen wir uns noch mit einem etwas komplizierteren Begriff für C-Klassen beschäftigen: ihre Größe bzw. Mächtigkeit. Definieren wir

$$
\mathrm{Cl} K, L \Rightarrow\left(K \cong L: \Leftrightarrow \bigvee_{F} F: K \rightarrow L \text { Bijektion }\right)
$$

so erhält man den Cantor'schen Satz:

$$
\text { (2.4.5) C. Cl } x \Rightarrow x \nRightarrow P_{c} x \text {. }
$$

Beweis 2.4.5: In der Sprache der modulierten Theorie ist (2.4.5) gerade der Cantor'sche Satz „Mg $x \Rightarrow x \not P x$ ”. (Man kann (2.4.5) aber auch direkt beweisen).

Somit haben wir in der C-Theorie auch eigentliche Klassen verschiedener "Größe" und es liegt daher nahe, den Begriff der Kardinalzahl auf C-Klassen zu erweitern, d.h. wir wollen jeder C-Klasse $x$ ein Element card $(x)$ zuordnen, so daß gilt:

$$
\text { C. } \mathrm{Cl} x, y \Rightarrow(\operatorname{card}(x)=\operatorname{card}(y) \Leftrightarrow x \cong y) \text {. }
$$

Das läuft aber gerade darauf hinaus, in der modulierten Theorie Kardinalzahlen (für Mengen) einzuführen. Dies erscheint ohne Ersetzungsaxiom aussichtslos, ist. aber unter Verwendung des Abschlußprinzips möglich. Etwas allgemeiner zeigen wir:

(2.4.6) Ist $\sim$ eine Äquivalenzrelation auf einer Klasse $K \subset \underline{\mathrm{A}}(\Omega)$, so gibt es eine Abbildung $F_{\sim}: K \rightarrow$ E mit $\bigwedge_{x, y \in K} F_{\sim}(x)=F_{\sim}(y) \Leftrightarrow x \sim y$.

Beweis (2.4.6): Wir definieren zuerst eine Abbildung $a: K \rightarrow \Omega$ durch $a(x):=\min \left\{a \mid[x] \cap \underline{\mathbf{A}}_{a} \neq \varnothing\right\}$, wobei $[x]$ die Äquivalenzklasse von $x \in K$ ist. Dann läßt sich $F_{\sim}: K \rightarrow E$ definieren durch $F_{\sim}(x):=\mathbf{e}\left([x] \cap \underline{\mathrm{A}}_{a(x)}\right)$ für $x \in K$, und man zeigt leicht für $x, y \in K: F_{\sim}(x)=F_{\sim}(y) \Leftrightarrow x \sim y$.

Zur Konstruktion der Kardinalzahlen von $\mathrm{C}$-Klassen betrachten wir auf E die Äquivalenzrelation $, x \sim y: \Leftrightarrow \mathbf{C} \cong \mathbf{C} y$ " und mit dem Abschlußprinzip AP kann man (2.4.6) anwenden. In analoger Weise lassen sich auch Ordinalzahlen für wohlgeordnete C-Klassen einführen.

\section{§ 3. Widerspruchsfreiheitsfragen}

3.0. Das Fundamentaxiom in der modulierten Theorie. Zur Motivation von Vereinigungs-, Potenz und Aussonderungsaxiom für C-Klassen haben wir die modulierte C-Theorie betrachtet. Um die Existenz nichttrivialer C-Klassen zu sichern, haben wir dann noch das Fundamentaxiom formuliert. Damit ist das Fundamentaxiom offenbar das stärkste der C-Klassen-Bildungsaxiome und es liegt daher nahe, die Auswirkungen dieses Axioms in der modulierten Theorie zu betrachten. Hierzu untersuchen wir zunächst einige modulierte Begriffe:

$$
\begin{gathered}
\underline{\mathbf{E}}_{c} \stackrel{c}{=} \underline{\mathbf{E}}, \\
\mathrm{El}_{c} x, y \Rightarrow(x, y)_{c} \stackrel{c}{=}(\mathbf{e} x, \mathbf{e} y),
\end{gathered}
$$

(3.0.2) für jede C-Formel $\mathfrak{N}(x, y)$ im engeren Sinne gilt: 
Auf die einfachen Beweise verzichten wir und wollen jetzt die Eigenschaften von $A_{0}=V(\varnothing)$ in der modulierten Theorie untersuchen. Da $A_{0}$ kein Urelement enthält, ist zu erwarten, $\mathrm{da} B$ sich viele Eigenschaften von $\underline{\mathrm{A}}_{0}$ in die modulierte Theorie übertragen:

$$
\begin{gathered}
x \in \in_{c} \underline{\mathrm{A}}_{0} \Leftrightarrow x \in \underline{\mathrm{A}}_{0}, \\
\text { (3.0.9) } \\
\text { (3.0.10) }
\end{gathered}
$$

Beweise (3.0.8)-(3.0.10): vgl. (2.1.15), (2.1.28), (2.1.30), (3.0.4), (3.0.6). Hieraus folgt mit (2.1.37) und (2.2.13).

(3.0.11) $\underline{\mathrm{A}}_{0}$ ist in der modulierten Theorie ein kleines inneres Modell.

Wie schon früher angedeutet, soll das Ersetzungsprinzip in der modulierten Theorie nicht gefordert werden (vgl. (2.1.37)), aber unter Verwendung von $\underline{A}_{0}$ kann man ein abgeschwãchtes Ersetzungsprinzip beweisen.

$$
x \in_{c} \underline{\mathrm{A}}_{0} \wedge f: x \rightarrow \underline{\mathrm{E}}_{c} \mathrm{Abb}_{c} \Rightarrow \mathrm{Mg}_{c} f[x]_{c} .
$$

Beweis (3.0.12): Nach (3.0.8) ist $x$ Menge und wegen (3.0.0), (3.0.4), (3.0.6) folgt $\operatorname{Mg} f[x]_{c}$.

Interessant ist nun, da $\beta$ man auch das Mengenprädikat „Mg” unter Verwendung von $\underline{\mathbf{A}}_{0}$ und des Auswahlprinzips $\mathbf{A M}$ in der modulierten Theorie charakterisieren kann:

$$
(3.0 .13)^{\mathbf{A M}} \quad \mathrm{Mg} x \Leftrightarrow \bigvee_{y \in \epsilon_{c} \mathbb{A}_{0}} x \cong_{c} y .
$$

Beweis (3.0.13):

$\Rightarrow$ "Wegen $\Omega \subset \underline{\mathrm{A}}_{0}$ und $\mathbf{A M}$ gibt es ein $y \in \underline{\mathrm{A}}_{0}$ mit $x \cong y$.

„ $\Leftarrow$ "Man hat mit (3.0.7) $\mathbf{C} \cong \cong \mathbf{C} y$, und da $y \in \underline{A}_{0}$ (vgl. 3.0.8) eine Menge ist, muß auch $C x$ und $x$ eine Menge sein.

Definiert man allgemein:

$$
x \in_{c} z: \Leftrightarrow \bigvee_{y \in} z \cong_{c} y
$$

so hat man etwas prägnanter:

$$
(3.0 .13)^{\mathbf{A M}} \quad \operatorname{Mg} x \Leftrightarrow x \underset{\sim}{\in_{c}} \underline{\mathrm{A}}_{0} .
$$

Unter Benutzung dieses Resultats sind wir nun in der Lage, für eine Klasse $K$ den Begriff des Fundaments von $K$ in der modulierten Theorie auszudrücken:

$$
\begin{aligned}
& (3.0 .15)^{\wedge M} \quad \mathrm{Fd} K \stackrel{c}{=}\left\{x \mid x \notin_{c} \underline{\mathrm{A}}_{0} \wedge \bigvee_{n \epsilon_{c} \omega_{c}\left(x_{0} \ldots x_{n}\right)_{c} \epsilon_{c} \underline{n}_{c}^{n+1}} x_{1}, \ldots, x_{n} \in_{\sim} \underline{\mathrm{A}}_{0} \wedge\right. \\
& \left.\wedge x \stackrel{c}{=} x_{0} \in_{c} x_{1} \in_{c} \ldots \in_{c} x_{n} \in_{c} K\right\}_{c} .
\end{aligned}
$$

Beweis (3.0.15): Für Elemente $x$ gilt

$$
\begin{aligned}
& x \in \mathrm{Fd} K \Leftrightarrow \mathrm{Ur} x \wedge \bigvee_{n \in \omega\left(x_{0}, \ldots, x_{n}\right) \in \mathrm{E}^{n+1}} x=x_{0} \in x_{1} \in \ldots \in x_{n} \in K \\
& \Leftrightarrow \neg \operatorname{Mg} x \wedge \bigvee_{n \in \omega} \bar{\bigvee}_{\left(x_{0}, \ldots, x_{n}\right) \in \mathbb{E}^{n+1}} \operatorname{Mg} x_{1}, \ldots, x_{n} \wedge x=x_{0} \in x_{1} \in \ldots \in x_{n} \in K
\end{aligned}
$$

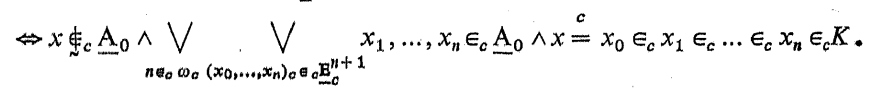

(Die letzte Äquivalenz folgt aus (3.0.13) und bisherigen Resultaten aus der modulierten Theorie). Damit ist $\mathrm{Fd} K=\left\{x \mid x \$_{c} \underline{\mathrm{A}}_{0} \wedge \ldots\right\}$ und mit (2.1.26) folgt (3.0.15).

Damit läßt sich das Fundamentaxiom in die modulierte Theorie formulieren;

$$
(3.0 .16)^{\mathrm{AM}} \quad \mathrm{Mg}_{c} \mathrm{Fd} x \Rightarrow \mathrm{Mg}_{c} x \text {. }
$$

Beweis (3.0.16): Nach (3.0.15) ist (3.0.16) von der Form $\mathfrak{A}_{c}$ für eine C-Formel $\mathfrak{A}$ und somit genügt es, wegen (2.1.16), (2.1.22) den Fall „Cl $x$ ” zu betrachten. Es gilt dann

$$
\mathrm{Mg}_{c} \mathrm{Fd} x \Rightarrow \mathrm{C}-\mathrm{ClFd} x \Rightarrow \mathrm{C}-\mathrm{Cl} x \Rightarrow \mathrm{Mg}_{c} x .
$$

Damit haben wir das Fundamentaxiom mit Hilfe von $\underline{A}_{0}$ in die modulierte Theorie „übersetzt”. Um die Rolle dieses Axioms in der modulierten Theorie weiterverfolgen zu können, „vergessen” wir die C-Theorie und betrachten im folgenden Abschnitt die modulierte Theorie als selbständige Theorie, d.h. als eine reine NBG-Theorie - ohne Ersetzungsaxiom - mit einem kleinen inneren Modell (als Analogon zu $\mathrm{A}_{0}$ ) und einem (3.0.16) entsprechenden Fundamentaxiom (mit einem (3.0.15) entsprechenden Fundamentbegriff).

3.1. Die modifizierte NBG-Theorie (M-Theorie). Entsprechend den Betrachtungen des vorigen Abschnitts, wollen wir jetzt die in 1.0 angegebene NGBTheorie leicht wie folgt modifizieren:

Modifikation 1: Das Rahmenaxiom R3 wird verschärft $\mathrm{zu}$

\section{$\mathbf{R 3}^{\prime}: \quad \mathrm{Cl} x$.}

Damit fallen die Begriffe Element und Menge zusammen und wir haben eine reine NBG-Theorie.

Modifikation 2: Eine Konstante $\underline{M}$ wird als neuer Grundbegriff mit folgenden Rahmenaxiomen eingeführt:

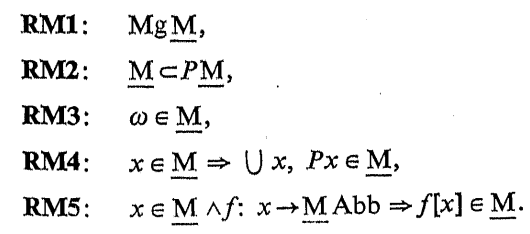


Die Rahmenaxiome besagen genau daß $\underline{M}$ ein kleines inneres Modell ist (vgl. (1.2.24) und (1.2.25)).

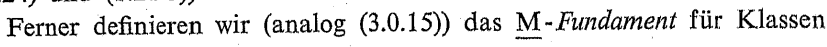

$$
\begin{gathered}
x \in z: \Leftrightarrow \bigvee_{y \in z} x \cong y, \\
\mathrm{Fd}_{\underline{M}} K:=\left\{x \mid x \notin \underline{\mathrm{M}} \wedge \bigvee_{n \in \omega} \bigvee_{\left(x_{0}, \ldots, x_{n}\right) \in \underline{\mathrm{Y}}^{n+1}} x_{1}, \ldots, x_{n} \in \underline{\mathrm{M}} \wedge\right. \\
\left.\wedge x=x_{0} \in x_{1} \in \ldots \in x_{n} \in K\right\}
\end{gathered}
$$

und fordern (analog (3.0.16)) das $\underline{\mathrm{M}}$-Fundamentaxiom

$$
\mathbf{F D}_{\underline{\mathbf{M}}}: \quad \mathrm{MgFd}_{\underline{\mathbf{M}}} K \Rightarrow \mathrm{Mg} K \text {. }
$$

Modifikation 3: Das Ersetzungsaxiom ERS für Mengen wird durch das folgende, wesentlich schwächere Ersetzungsprinzip ersetzt:

ERSM: $\quad x \in \underline{\mathrm{M}} \wedge f: x \rightarrow \underline{\mathrm{V} A b b} \Rightarrow \operatorname{Mg} f[x]$.

Die durch die Modifikationen 1-3 bestimmte (reine) NBG-Theorie bezeichnen wir im folgenden kurz als die $\mathrm{M}$-Theorie.

Bevor wir die $\mathrm{M}$-Theorie weiter untersuchen, stellen wir fest, daß vermöge der Interpretation von $\epsilon, \mathrm{El}, \mathrm{Cl}, \mathrm{M}$ durch $\epsilon_{c}, \mathrm{El}_{c}, \mathrm{Cl}_{c}, \mathrm{~A}_{0}$ im Rahmen der $C$-Theorie die modulierte C-Theorie ein "Modell" $\operatorname{der} \mathrm{M}$-Theorie bildet, denn in 2.1 und 3.0 wurde die Gültigkeit der Axiome der $\underline{M}$-Theorie bei dieser Interpretation bereits festgestellt. Folglich haben wir das

Widerspruchsfreiheitstheorem 1. Die $\mathrm{M}$-Theorie ist relativ wiederspruchsfrei zur C-Theorie.

Die Umkehrung dieses Theorems, daß auch die C-Theorie relativ wiederspruchsfrei zur $\mathrm{M}$-Theorie ist, zeigen wir nach einige Vorbereitungen in 3.3. Insbesondere benötigen wir dafür noch ein schwaches Auswahlprinzip, das wir schon hier formulieren, ohne es als Axiom zu fordern (jeder Gebrauch dieses Prinzips wird explizit gekennzeichnet).

M-Auswahlprinzip:

MA: $\quad x \in \underline{\mathrm{M}} \wedge \bigwedge_{y \in x}(y \in \underline{\mathrm{M}} \wedge y \neq \emptyset) \Rightarrow \bigvee_{f: x \rightarrow \cup x} \bigwedge_{y \in x} f(y) \in y$.

Als Ergänzung zum Widerspruchsfreiheitstheorem 1 formulieren wir das

Widerspruchsfreiheitstheorem 2. Die $\mathrm{M}$-Theorie mit M-Auswahlprinzip MA ist relativ widerspruchsfrei zur C-Theorie mit Auswahlprinzip AM für Mengen.

Beweis: Wir verwenden wieder die Interpretation $\epsilon_{c}, \mathrm{El}_{c}, \mathrm{Cl}_{c}, \mathrm{~A}_{0}$ für die Grundbegriffe $\in, \mathbf{C l}, \mathrm{El}, \underline{\mathbf{M}}$ und müssen nur noch zeigen, daß $\underline{\mathbf{M A}}$ erfülltt ist, d.h.

$$
x \in_{c} \underline{\mathrm{A}}_{0} \wedge \bigwedge_{y \epsilon_{c} x}\left(y \Theta_{c} \underline{\mathrm{A}}_{0} \wedge y_{c} \neq{ }_{c} \varnothing_{c}\right) \Rightarrow \bigvee_{f: x \rightarrow \cup_{c} x \mathrm{Abb}_{c}} \bigwedge_{y \epsilon_{c} x} f(y)_{c} \in_{c} y .
$$

Die Gültigkeit dieser Formel erkennt man (unter Benutzung von AM) leicht mit (3.0.13) und weiteren Resultaten aus 2.1 und 3.0.

Abschließend beweisen wir noch, daß in der M-Theorie ohne M-Fundamentaxiom das M-Fundamentaxiom aus dem Ersetzungsprinzip für Mengen und dem Fundierungsprinzip folgt. Insbesondere ist dann die M-Theorie relativ widerspruchsfrei zur reinen NBG-Theorie mit Fundierungsprinzip und einem kleinen inneren Modell.

\section{In der $\mathrm{M}$-Theorie ohne $\mathbf{F D}_{\mathrm{M}}$ gilt:}

$$
\text { FUND } \wedge \text { ERS } \Rightarrow \text { FD }_{\underline{M}} \text {. }
$$

Beweis (3.1.2): Es sei $K$ eine Klasse, deren $\underline{\mathrm{M}}$-Fundament $\mathrm{Fd}_{\underline{M}} K$ eine Menge ist. Unter Verwendung des Ersetzungsprinzips definieren wir rekursiv eine Menge $L$ und zeigen dann mit dem Fundierungsprinzip durch Induktion über $\epsilon$, daß $K \subset L$ gilt. Nach dem Aussonderungsaxiom ist $K$ dann eine Menge.

Für $m:=\mathrm{M} \cap \Omega$ ist $m \in \Omega$ eine Limeszahl. Die Abbildung $F: m \rightarrow \underline{V}$ wird rekursiv (unter Verwendung von ERSM) definiert durch:

$$
F(a):=\mathrm{Fd}_{M} K \cup \underset{b<a}{\bigcup} P F(b) \text {. }
$$

Dann ist $L:=\bigcup_{a<m} F(a)$ nach dem Ersetzungsprinzip eine Menge. Durch $\in$-Induktion zeigen wir jetzt: $y \in K \Rightarrow y \in L$.

Fall 1: $y \underset{\sim}{\ddagger}$. Dann ist $y \in \mathrm{Fd}_{M} K=F(0) \subset L$.

Fall 2: $y \in \underline{\sim}$. Dann gibt es ein $x \in \underline{M}$ und eine Bijektion $f: x \rightarrow y$. Da $y \subset L$ nach Induktionsvoraussetzung gilt, läßt sich eine „Rangabbildung” $r: y \rightarrow m$ definieren durch $r(z):=\min \{a \mid z \in F(a)\}$. Insgesamt haben wir also eine Abbildung $\underset{x \rightarrow y \rightarrow m}{\rightarrow} m$.

Da $M$ inneres Modell und $m$ gerade die Klasse der Ordinalzahlen in $\underline{M}$ ist, hat das Bild von $r f$ eine obere Schranke, d.h. es gibt ein $a<m$ mit $r f(u) \leqslant a$ für alle $u \in x$. Damit ist auch $r(z) \leqslant a$ bzw. $z \in F(a)$ für jedes $z \in y$. Also ist $y \subset F(a)$, und da $m$ Limeszahl ist, folgt $a+1<m$ und $y \in F(a+1) \subset L$.

Es sei noch erwähnt, daß die Widerspruchsfreiheitsresultate erhalten bleiben, wenn man zur $\mathbf{C}$ - und $M$-Theorie das starke Klassenkomprehensionsaxiom KOMPS und bzw. oder die Ersetzungsprinzipien ERSC und ERS hinzufügt, weil diese ihre Modulationen implizieren.

3.2. Innere Modelle in der M-Theorie. In diesem Abschnitt sollen einige Vorbereitungen zum Beweis der Widerspruchsfreiheit der C-Theorie relativ zur $\underline{M}$ Theorie, den wir unter Verwendung innerer Modelle führen werden, getroffen werden. Da die C-Theorie Urelemente hat, die M-Theorie aber nicht, kommen daher für den Widerspruchsfreiheitsbeweis nur nicht-normale innere Modelle in- 
frage. Wir wollen daher einige nicht-normale innere Modelle konstruieren. Definiert man

$$
m:=\underline{\mathrm{M}} \cap \Omega
$$

so gilt (wie bereits im Beweis von (3.1.2) ausgeführt):

$$
\begin{gathered}
m \in \Omega \text { Limeszahl, } \\
x \in \underset{\sim}{\underline{M}} \wedge f: x \rightarrow m \Rightarrow \bigvee_{a<m} \bigwedge_{y \in x} f(y) \leqslant a .
\end{gathered}
$$

Zunächst wollen wir jetzt zeigen, daß es zu jeder Teilmenge von $\{m\} \times \underline{V}$ ein kleines inneres Modell gibt, dessen Urelementklasse gerade diese Teilmenge ist. Im folgenden betrachten wir also eine feste Teilmenge $L \subset\{m\} \times \underline{\mathrm{V}}$. Zur Abkürzung setzen wir

$$
\underline{\mathrm{M}}_{\epsilon}:=\{x \mid x \underset{\sim}{\in} \underline{\mathrm{M}}\}
$$

und definieren jetzt die Konstruktionsabbildung $\underline{\mathrm{K}}^{\mathrm{L}}: m \rightarrow \underline{\mathrm{V}}$ rekursiv durch

$$
\bigwedge_{a<m} \underline{\mathrm{K}}_{a}^{L}:=\bigcup_{b<a}\left(L \cup\left(\underline{\mathrm{M}}_{\tilde{e}} \cap P \underline{\mathrm{K}}_{b}^{L}\right)\right) .
$$

Erweitern wir (3.2.4) noch durch

$$
\underline{\mathrm{K}}(L):=\underline{\mathrm{K}}_{m}^{L}:=\bigcup_{a<m} \underline{\mathrm{K}}_{a}^{L}
$$

so gilt wegen (3.2.1):

$$
\left.\underline{\mathrm{K}}_{m}^{L}=\underset{b<m}{\bigcup}\left(L \cup \underset{\underline{\mathrm{M}}_{\epsilon}}{\mathrm{G}} \cap P \underline{\mathrm{K}}_{b}^{L}\right)\right) .
$$

Es soll nun gezeigt werden, da $\underline{\mathrm{K}}(L)$ ein kleines inneres Modell mit $L$ als Urelementklasse (d.h. $\underline{\mathrm{K}}(L) \backslash P \underline{\mathrm{K}}(L)=\bar{L}$ ) ist. Dies erfolgt in drei Schritten, indem wir zeigen:

(1) $\underline{\mathrm{K}}(L)$ ist eine Menge (das folgt nicht aus dem Ersetzungsprinzip ERSM).

(2) $\underline{\mathrm{K}}(L) \backslash P \underline{\mathrm{K}}(L)=L$.

(3) $\underline{\mathrm{K}}(L)$ ist inneres Modell.

Im ersten Schritt zeigen wir, daß das $\underline{M}$-Fundament von $\underline{K}(L)$ im $\underline{M}$-Fundament von $L$ liegt (das eine Menge ist) und erhalten dann (1) mit dem $\overline{\mathrm{M}}$-Fundamentaxiom

$$
\begin{gathered}
\mathrm{Fd}_{\underline{M}}\left(\mathrm{M}_{\tilde{\Xi}} \cap P x\right) \subset \mathrm{Fd}_{\underline{M}} x, \\
\mathrm{Fd}_{\underline{M}} \cup x \subset \bigcup_{y \in x} \mathrm{Fd}_{\underline{M}} y, \\
\mathrm{Fd}_{\underline{M}}(x \cup y)=\mathrm{Fd}_{\underline{M}} x \cup \mathrm{Fd}_{\underline{M}} y, \\
\mathrm{Mg} x \Rightarrow \operatorname{MgFd}_{\underline{M}} x, \\
a \leqslant m \Rightarrow \mathrm{Fd}_{\underline{M}} \underline{\mathrm{K}}_{a}^{L} \subset \mathrm{Fd}_{\underline{M}} L, \\
\operatorname{Mg}_{\underline{K}}(L) .
\end{gathered}
$$

Beweise (3.2.7)-(3.2.9) folgen unmittelbar aus der Definition des $\underline{M}$-Fundaments.

Beweis (3.2.10): Mit ERSM kann man $\mathrm{Fd}_{M} x$ analog (1.1.1)-(1.1.5) konstruieren.

Beweis (3.2.11) folgt durch Induktion über $a$ aus (3.2.7)-(3.2.9):

$$
\begin{aligned}
& \mathrm{Fd}_{\underline{M}} \mathrm{~K}_{a}^{L}=\mathrm{Fd}_{\underline{M}} \underset{b<a}{\cup}\left(L \cup\left(\underline{\mathrm{M}}_{\boldsymbol{\sim}} \cap P \underline{\mathrm{K}}_{b}^{L}\right)\right) \\
& \subset \mathrm{Fd}_{\underline{M}}\left(L \cup \underset{b<a}{\cup}\left(\underline{\mathrm{M}}_{\boldsymbol{\sigma}} \cap P \underline{\mathrm{K}}_{b}^{L}\right)\right) \\
& \left.=\mathrm{Fd}_{\underline{M}} L \cup \mathrm{Fd}_{\underline{M}_{b<a}} \cup\left(\underline{\mathrm{M}}_{\mathrm{e}} \cap P \underline{\mathrm{K}}_{b}^{L}\right)\right) \\
& \subset \mathrm{Fd}_{\mathrm{M}} L \cup \mathrm{Fd}_{\mathrm{M}} L \text { (nach Induktionsvoraussetzung). }
\end{aligned}
$$

Beweis (3.2.12) vgl. (3.2.10) und (3.2.11). Damit kommen wir zum zweiten Schritt, bei dem wir wesentlich von der Inklusion $L \subset\{m\} \times \underline{\mathrm{V}}$ Gebrauch machen, wir zeigen:

$$
\begin{gathered}
m \cap(\{m\} \times \underline{\mathrm{V}})=\varnothing \wedge m \notin\{m\} \times \underline{\mathrm{V}}, \\
a \leqslant m \Rightarrow a \subset \underline{\mathrm{K}}_{a}^{L} \backslash L \wedge a \notin \underline{\mathrm{K}}_{a}^{L}, \\
a \leqslant m \Rightarrow(\{m\} \times \underline{\mathrm{V}}) \cap P \underline{\mathrm{K}}_{a}^{L}=\varnothing, \\
L \cap \underline{\mathrm{P}} K(L)=\varnothing, \\
\underline{\mathrm{K}}(L) \backslash P \underline{\mathrm{K}}(L)=L .
\end{gathered}
$$

Beweis (3.2.13): Für jede Menge $x$ ist $m \in\{m\} \in(\{m\},\{m, x\})=(m, x)$ und somit $(m, x) \notin m$ und $(m, x) \neq m$ wegen $m \in \Omega$.

Beweis (3.2.14): Indukțion über $a$. Für $b<a$ bzw. $b \in a$ ist nach Induktionsvoraussetzung $b \subset \mathrm{K}_{b}^{L}$, also $b \in M_{\mathbb{e}} \cap P \underline{\mathrm{K}}_{b}^{L} \subset \underline{\mathrm{K}}_{a}^{L}$, und somit folgt $a \subset \underline{\mathrm{K}}_{a}^{L}$ und nach (3.2.13) sogar $a \subset \bar{K}_{a}^{L} \backslash L$, weil $L \subset \tilde{\{}\{m\} \times \bar{V}$. Angenommen, es ist $a \in \underline{K}_{a}^{L}$, also nach (3.2.13) sogar $a \in \overline{\mathrm{K}}_{a}^{L} \backslash L$, so gibt es nach (3.2.4)-(3.2.6) ein $b<a$ mit $a \subset \underline{\mathrm{K}}_{b}^{L}$ und somit $b \in \mathrm{K}_{b}^{L}$ im Widerspruch zur Induktionsvoraussetzung.

Beweis (3.2.15): Angenommen, für eine Menge $x$ ist $(m, x)=(\{m\},\{m, x\})$ $\in P \mathrm{~K}_{a}^{L}$, also $\{m\} \in \mathrm{K}_{a}^{L}$ und wegen $\{m\} \notin L \subset\{m\} \times \underline{\mathrm{V}}$ sogar $\{m\} \in \mathrm{K}_{a}^{L} \backslash L$. Nach (3.2.4)-(3.2.6) gibt es dann ein $b<a$ mit $\{m\} \in P \underline{K}_{b}^{L}$, also $m \in \underline{K}_{b}^{L} \subset \underline{K}_{m}^{\bar{L}}$ im Widerspruch zu 3.2.14.

Beweis (3.2.16) folgt aus (3.2.15) für $a=m$.

Beweis (3.2.17) folgt aus (3.2.16).

Jetzt wird im dritten Schritt gezeigt, daß $\underline{K}(L)$ ein inneres Modell ist. Es werden einige Hilfssätze über $\underline{M}_{a}$ bewiesen und dann wenden wir das Kriterium (1.2.24) an.

$$
\begin{gathered}
x \in \underline{\underline{M}} \Rightarrow p x \in \underset{\sim}{\in \underline{M}}, \\
f: x \rightarrow y \text { injektiv } \wedge y \underset{\sim}{\in \underline{M}} \Rightarrow x \in \underline{\underline{M}},
\end{gathered}
$$



$(3.2 .21)^{\mathrm{MA}}$ $f: x \rightarrow y$ subjektiv $\wedge x \underset{\sim}{\in \underline{M}} \Rightarrow y \underset{\sim}{\in \underline{M}}$,

$$
x \underset{\sim}{\in} \underline{M} \wedge x \subset \underline{M}_{\in} \Rightarrow \bigcup x \underset{\sim}{\in} \underline{M} .
$$

Beweis (3.2.18): $\mathrm{Zu} x \in \underline{\mathrm{M}}$ gibt es ein $y \in \underline{\mathrm{M}}$ mit $x \cong y$. Dann ist auch $P y \in \mathrm{M}$ und $P x \cong P y$, also $P_{x} \in \mathrm{M}$.

Beweis (3.2.19): Es gibt ein $z \in M$ mit $y \cong z$. Wegen $x \cong f[x] \subset y$ gibt cs cin $u \subset z$ mit $x \cong u$, also $u \in \mathrm{M}$ und damit $x \in M$.

Beweis (3.2.20): Es ist bekanntlich $y \cong\left\{f^{-1}[\{v\}] \mid v \in y\right\} \subset P x$. Nach (3.2.18) ist $P x \underset{\sim}{\in} \underline{\mathrm{M}}$ und nach (3.2.19) folgt $y \underset{\sim}{\in} \underline{\mathrm{M}}$.

Beweis (3.3.21): Zu jedem $y \in x$ gibt es nach MA ein $b \in m$ mit $y \cong b$, also gibt es eine Abbildung $h: x \rightarrow m$ mit $h(y)=\min \{\bar{x} \mid y \cong b\} \cong y$. Jetzt wenden wir MA auf $\{\operatorname{Bij}(h(y), y) \mid y \in x\}=x$ an - hierbei sei $\operatorname{Bij}(L, N)$ die Menge der Bijektionen $L \rightarrow N$ für Mengen $L, N$-denn für jedes $y \in x$ ist $\operatorname{Bij}(h(y), y) \neq \varnothing$ und $\operatorname{Bij}(h(y), y) \cong \operatorname{Bij}(h(y), h(y)) \in M$. Somit haben wir eine Abbildung $g: x \rightarrow \underline{V}$ mit $g(y): h(y) \rightarrow y$ Bijektion für jedes $y \in x$. Dann können wir eine Subjektion $\dot{U} h(y) \rightarrow \cup x$ definieren - hierbei bezeichnet $\dot{U}$ die disjunkte Vereinigung - die $y \in x$

auf jedem $h(y)$ mit $g(y)$ übereinstimmt. Nun ist $\bigcup_{y \in x} h(y) \in \underline{\mathrm{M}}$ wegen $h(y) \in \underline{\mathrm{M}}$ und $x \underset{\sim}{\underline{M}}$ (in $\underline{M}$ gilt ERS). Mit (3.2.20) folgt $U \underset{\sim}{x \in M}$.

Jetzt sind wir in der Lage, zu zeigen, daß $\underline{\mathrm{K}}(L)$ ein inneres Modell ist:

$$
\underline{\mathrm{K}}(L) \backslash L=\underline{\mathrm{M}}_{\tilde{\epsilon}} \cap P \underline{\mathrm{K}}(L)=\underline{\mathrm{K}}(L) \cap P \underline{\mathrm{K}}(L),
$$

(3.2.23) $)^{\mathbf{M A}} \quad \underline{\mathrm{K}}(L)$ ist inneres Modell mit Auswahlaxiom $\mathbf{A M}_{\mathbf{K}(L)}$.

Beweis 3.2.22: Die Inklusionen $\underline{\mathrm{K}}(L) \cap P \underline{\mathrm{K}}(L) \subset \underline{\mathrm{K}}(L) \backslash L \subset \underline{\mathrm{M}_{\epsilon}} \cap P \underline{\mathrm{K}}(L)$ folgen aus (3.2.16), (3.2.5) und (3.2.6). $\overline{\mathrm{Zu}}$ zeigen ist noch: $\underline{\mathrm{M}}_{\epsilon} \bar{\cap} P \underline{\mathrm{K}}(\bar{L}) \subset \underline{\mathrm{K}}(L) \cap$ $\cap P \underline{\mathrm{K}}(L)$. Sei also $x \subset \underline{\mathrm{K}}(L)$ und $x \in \underline{\mathrm{M}}$. Für die „Rangabbildung" $r: x \rightarrow m$, definiert durch $r(y)=\min \left\{a \mid \bar{y} \in \mathrm{K}_{a}^{L}\right\}$, gibt es nach (3.2.2) ein $a<m$ mit $r(y) \leqslant a$ bzw. $y \in \underline{\mathrm{K}}_{a}^{L}$ für alle $y \in x$. Dann ist aber $x \subset \underline{K}_{a}^{L}$, also $x \in \underline{\mathrm{M}}_{\epsilon} \cap P \underline{K}_{a}^{L} \subset \underline{\mathrm{K}}(L)$. Den Beweis von (3.2.23) können wir mit (3.2.22) auf den wesentlich allgemeinen Satz zurückm führen.

(3.2.24) $\underline{\underline{M A}} \quad K \cap P K=\underline{\mathrm{M}_{\epsilon}} \cap P K \Rightarrow K$ ist inneres Modell mit Auswahlaxiom $\mathbf{A M}_{K}$.

Beweis 3.2.24: Wir wenden das Kriterium (1.2.24) an. Durch Induktion zeigen wir $a<m \Rightarrow a \in K \cap P K$, woraus insbesondere $\omega \in K \cap P K$ folgt. Nach Induktionsvorausserzung ist $a \subset K$ und somit $a \in \underline{\mathrm{M}}_{\S} \cap P K$. Sei nun $x \in K \cap P K$ d.h. $x \underset{\sim}{\subseteq}$ und $x \subset K$. Für $y \subset x$ folgt $y \subset K$ und $\bar{y} \tilde{\epsilon} \mathrm{M}$ (3.2.19), also $y \in \underline{\mathrm{M}}_{\mathrm{a}} \cap P K \subset K$,

Damit ist $P x \subset K$ und $P x \in \mathrm{M}$ (3.2.16), also $P x \in K \cap P K$. Für eine Abbildung $f: x \rightarrow K$ ist $f[x] \in P K$ (nach E्ERSM!) und $f[x] \in \underline{M}$ (3.2.20), also $f[x] \in K \cap P K$, Gilt schließlich $x \subset P K$ so ist für jedes $y \in x$ auch $\tilde{y} \in K \cap P K$, also $y \in \mathrm{M}$ und $y \subset K$. Folglich ist $U x \in \underset{\sim}{\underline{M}}\left(3.2 .21^{\mathbf{M A}}\right)$ und $U x \in K$, also $U x \in K \cap P K$.
Folglich ist $K$ ein inneres Modell. Es bleibt die Gültigkeit von $\mathbf{A M}_{K}$ zu zeigen, die jedoch leicht aus $\underline{M} \mathbf{A}$ und $K \cap P K \subset \underline{M}_{\sim}$ folgt.

Abschließend zeigen wir noch, daß in dem Modell $\underline{\mathrm{K}}(L)$ auch das Fundierungsprinzip gilt:

Definiert man die Rangabbildung $r: \underline{\mathrm{K}}(L) \rightarrow m$ durch

$$
\widehat{\widehat{N}}_{x \in \mathbb{K}(L)} r(x):=\min \left\{a \mid x \in \underline{\mathbb{K}}_{a}^{L}\right\},
$$

so gilt

$$
\bigwedge_{x, y \in \mathbb{K}(L) \backslash L}(x \in y \Rightarrow r(x)<r(y)) .
$$

In $K(L)$ gilt das Fundierungsprinzip (d.h. $\mathrm{FUND}_{\mathrm{K}(L)}$ ).

Beweis (3.2.25): Wegen $y \in \underline{K}_{r(y)}^{L} \backslash L$ gibt es ein $a<r(y)$ mit $y \subset \underline{K}_{a}^{L}$, also $x \in \underline{\mathrm{K}}_{a}^{L}$ und somit $r(x) \leqslant a<r(y)$.

Beweis (3.2.26): Die Behauptung folgt leicht aus (3.2.25).

3.3 Die relative Widerspruchsfreiheit der C-Theorie. In diesem Abschnitt wollen wir das Widerspruchsffeiheitstheorem 2 verschärfen zu

Widerspruchsfreiheitstheorem 3. Die C-Theorie mit dem Auswahlprinzip AM für. Mengen ist genau dann widerspruchsfrei, wenn die $\underline{\mathrm{M}}$-Theorie mit dem $\underline{\mathrm{M}}$-Auswahlprinzip MA widerspruchsfrei ist.

Es ist also zu zeigen, daß die C-Theorie mit $\mathbf{A M}$ relativ widerspruchsfrei zur M-Theorie mit MA ist. Fierzu geben wir eine Klasse $K$ an, die Voraussetzung von (3.2.24) erfüllt und zeigen, daß bei einer geeigneten Interpretation von $C, K$ sogar ein Modell der C-Theorie ist. Die Idee, welche der Konstruktion von $K$ zugrunde liegt, geben wir kurz an. Mit $\underline{M}$ haben wir ein kleines inneres Modell (der reinen NBG-Theorie), in der die Elemente von $P$ M $\backslash \mathrm{M}$ gerade die eigentlichen Klassen sind. Betrachten wir nun die Abbildung $(m,-): P \mathrm{M} \backslash \underline{\mathrm{M}} \rightarrow\{m\} \times \underline{\mathrm{V}}$, so haben wir mit $\mathrm{K}(\{m\} \times(P \mathrm{M} \backslash \mathrm{M}))$ wieder ein kleines inneres Modell (einer NBGTheorie mit Urelementen), dessen Urelemente gerade den eigentlichen Klassen von $M$ entsprechen und deren eigentliche Klassen jetzt die Elemente von $P \mathrm{~K}(\{m\} \times(P \mathrm{M} \backslash \mathrm{M})) \backslash \mathrm{K}(\{\dot{m}\} \times(F \mathrm{M} \backslash \mathrm{M}))$ sind. Dieses Verfahren zur Gewinnung neuer Modelle mit jeweils mehr Urelementen läßt sich iterieren. Bezeichnen wir die Abbildung $(m,-): V \rightarrow\{m\} \times V$ kurz mit. e, so läßt sich eine Folge kleiner innerer Modelle definieren durch:

$$
A_{0}:=\underline{\mathrm{M}}, \quad A_{a+1}:=\underline{\mathrm{K}}\left(\mathrm{e}\left[P A_{a} \backslash A_{a}\right]\right) .
$$

Die "Urelemente" von $A_{u+1}$ entsprechen vermöge e gerade den „eigentlichen Klassen" von $A_{a}$. Wir setzen die Konstruktion der Modelle noch auf ganz $m$ fort, durch

$$
A_{a}:=\underline{\mathrm{K}}\left(\bigcup_{b<a} e\left[P A_{b} \backslash A_{b}\right]\right) \quad \text { für Limeszahlen } a<m .
$$


Dann ist $A_{m}:=\bigcup_{a<m} A_{a}$ das gesuchte Modell, wobei die Interpretation von $\mathbf{C}$ im wesentlichen durch die Umkehrabbildung $\mathrm{e}^{-1}$ gegeben ist.

Nach diesen Erörterungen kommen wir zum formalen Beweis. Aus suggestiven Gründen definieren wir:

(3.3.0) Die Abbildung $\mathrm{e}: \underline{\mathrm{V}} \rightarrow\{m\} \times \underline{\mathrm{V}}$ sei definiert durch

$$
\bigwedge_{\operatorname{Mg} x} \mathrm{e} x=(m, x) \text {. }
$$

Jetzt wird die Familie $\left(A_{a}\right)_{a<m}$ definiert

(3.3.1) Die Abbildung $A: m \rightarrow \underline{\mathrm{V}}$ wird rekursiv definiert durch:

$$
\bigwedge_{a<m} A_{a}:=\mathrm{K}\left(\bigcup_{b<a} \mathrm{e}\left[P A_{b} \backslash A_{b}\right]\right)
$$

Zur Abkürzung setzen wir gleich:

$$
\begin{aligned}
\bigwedge_{a<m} B_{a} & :=\bigcup_{b<a} \mathrm{e}\left[P A_{b} \backslash A_{b}\right] \\
A_{m} & :=\bigcup_{a<m} A_{a} \\
B_{m} & :=\bigcup_{a<m} B_{a}=\bigcup_{a<m} \mathrm{e}\left[P A_{a} \backslash A_{a}\right] .
\end{aligned}
$$

Also ist

$$
\begin{gathered}
a<m \Rightarrow A_{a}=\underline{\mathrm{K}}\left(B_{a}\right), \\
a \leqslant b \leqslant m \Rightarrow A_{a} \subset A_{b} \wedge B_{a} \subset B_{b} .
\end{gathered}
$$

$A_{m}$ soll nun das gesuchte Modell werden. Bevor wir C übet $A_{m}$ interpretieren, zeigen wir, daß $A_{m}$ ein inneres Modell ist, dessen Klasse von Urelementen gerade $B_{m}$ ist.

$$
\begin{gathered}
A_{m} \backslash P A_{m}=B_{m}, \\
A_{m} \cap P A_{m}=\underline{M}_{\sim} \cap P A_{m}=\bigcup_{a \in m}\left(A_{a} \backslash P A_{a}\right),
\end{gathered}
$$

(3.3.9) $)^{\mathrm{MA}} \quad A_{m}$ ist inneres (NBG-) Modell mit Auswahlaxiom AM.

Beweis (3.3.7): Mit (3.2.17) hat man

$$
A_{m} \backslash P A_{m}=\bigcup_{a<m} A_{a} \backslash P \underset{a<m}{\bigcup} A_{a} \subset \bigcup_{a<m}\left(A_{a} \backslash P A_{a}\right)=\bigcup_{a<m} B_{a}=B_{n} .
$$

Es bleibt noch zu zeigen $B_{m} \cap P A_{m}=\varnothing$, und wir beweisen allgemeiner $(\{m\} \times V) \cap$ $\cap P A_{m}=\varnothing$. Angenommen, für eine Menge $x$ ist $\{\{m\},\{m, x\}\}=(m, x) \in P A_{m}$, also $\{m\} \in A_{m}$. Dann gibt es ein $a<m$ mit $\{m\} \in A_{a}$ und somit $(m, m)=\{\{m\}\}$ $\in P A_{a}$ im Widerspruch zu (3.2.15) (für $a=m, L=B_{a}$ ).

Beweis (3.3.8): Mit (3.3.7) und (3.2.22) hat man

$$
\begin{aligned}
A_{m} \cap P A_{m} \subset A_{m} \backslash B_{m} \subset \bigcup_{a<m} A_{a} \backslash \bigcup_{a<m} B_{a} \subset \bigcup_{a<m}\left(A_{a} \backslash B_{a}\right) \\
=\bigcup_{a<m}\left(A_{a} \backslash P A_{a}\right)=\bigcup_{a<m}\left(\underline{M}_{\tilde{a}} \cap P A_{a}\right) \subset \underline{\sim}_{a} \cap P A_{m} .
\end{aligned}
$$

Da trivialerweise $\bigcup_{a<m}\left(A_{a} \cap P A_{a}\right) \subset A_{m} \cap P A_{m}$ gilt, bleibt nur noch zu zeigen: $\underline{\mathrm{M}}_{a_{a}} \cap P A_{m} \subset \bigcup_{a<m}\left(A_{a} \cap P A_{a}\right)$. Sei also $x \in \underset{\sim}{\underline{M}}$ und $x \subset A_{m}$. Definiert man die „Rangabbildung" $r: x \rightarrow m$ durch $r(y)=\min \left\{a \mid y \in A_{a}\right\}$, so gibt es nach (3.2.2) ein $a<m$ mit $r(y) \leqslant a$ bzw. $y \in A_{a}$ für alle $y \in x$. Also ist $x \in \underset{\sim}{\mathbb{M}_{\epsilon}} \cap P A_{a}=A_{a} \cap P A_{a}$ (3.2.22).

Beweis (3.3.9) folgt aus 3.2.24 ${ }^{\mathrm{MA}}$ mit (3.3.8).

(3.3.7) and (3.3.8) besagen gerade, daß die Klasse der Urelemente (bzw. Mengen) in $A_{m}$ genau die Vereinigung der Klassen der Urelemente (bzw. Mengen) in den $\boldsymbol{A}_{a}$ 's sind. Speziell sind die eigentlichen Klassen in den $A_{a}$ 's auch eigentliche Klassen in $A_{m}$ :

$$
a \leqslant m \Rightarrow P A_{a} \backslash A_{a} \subset P A_{m} \backslash A_{m} .
$$

Beweis (3.3.10). Wegen (3.3.8) und (3.2.22) ist: $A_{m} \cap P A_{a} \subset \underline{\mathrm{M}}_{\underline{\sim}} \cap P A_{a}$ $=A_{a} \cap P A_{a}$, woraus die Behauptung folgt.

Jetzt soll der Grundbegriff $\mathbf{C}$ in $A_{m}$ interpretiert werden. Hierzu definieren wir zunächst:

$$
\begin{aligned}
x \in B_{m} & \Rightarrow \mathfrak{C} x:=\mathrm{e}^{-1} x, \\
x \in A_{m} \backslash B_{m} \Rightarrow \mathfrak{C} x: & =x
\end{aligned}
$$

und beweisen

$$
\begin{array}{r}
A_{m} \backslash B_{m}=A_{m} \cap P A_{m}=\bigcup_{a<m}\left(A_{a} \backslash B_{a}\right), \\
x \in B_{m} \Rightarrow \mathfrak{C} x \in P A_{m} \backslash A_{m}, \\
a<m \wedge x \in A_{a} \Rightarrow \mathfrak{C} x \subset A_{a}, \\
a<m \wedge x \subset A_{a} \Rightarrow \underset{y \in A_{a+1}}{\bigvee} x=\mathfrak{C} y,
\end{array}
$$

Beweis (3.3.13): vgl. Beweis (3.3.8).

Beweis (3.3.14): vgl. (3.3.4) und (3.3.13).

Beweis (3.3.15): Für $x \in B_{a}$ gibt es ein $b<a$ mit $\mathbb{C} x \in P A_{b}$, also $\mathbb{C} x \subset A_{b} \subset A_{a}$, und für $x \notin B_{a}$ ist $\mathfrak{C} x=x\left((3.3 .12)\right.$ und (3.3.13)) und $x \in P A_{a}$ (3.2.22). 
Beweis (3.3.16). Für $x \in A_{a}$ ist $x \in A_{a} \cap P A_{a}=A_{a} \backslash B_{a} \subset A_{m} \backslash B_{m}$ ((3.2.22), (3.3.13)), also $\mathfrak{C} x=x$. Und für $x \notin A_{a}$ ist $x \in P A_{a} \backslash A_{a}$, also $\mathrm{e} x \in B_{a+1} \subset A_{a}$ mit cex $=x$.

Nun wird die formale Interpretation von $\mathbf{C}$ in $A_{m}$ definiert.

$$
\text { (3.3.17) } \quad \mathrm{C}_{A_{m}}:=\left\{(x, y) \mid\left(x \in B_{m} \wedge y \in \mathrm{e}^{-1} x\right) \vee\left(x \in A_{m} \backslash B_{m} \wedge y \in x\right)\right\} \text {. }
$$

Es folgt sofort:

$$
\begin{gathered}
\mathrm{C}_{A_{m}} \subset A_{m} \times A_{m}, \\
(3.3 .19) \quad x \in A_{m} \Rightarrow \mathrm{C}_{A_{m}}(x)=\mathbb{C} x
\end{gathered}
$$

Es gelten dann die Relativierungen der Rahmenaxiome R4-R7

$$
(3.3 .20) \quad(\mathbf{R} 4 \wedge \mathbf{R 5} \wedge \mathbf{R 6} \wedge \mathbf{R} 7)_{A_{m}} \text {. }
$$

Beweis (3.3.20): Mit (3.3.19) erhält man die Behauptungen aus (3.3.18) (R4), (3.3.11), (3.3.12) (R5), (3.3.14) (R6) und (3.3.12), (3.3.13) (R7).

Zum Nachweis der Relativierung der C-Klassenbildungsaxiome stellen wir fest:

$$
x \subset A_{m} \Rightarrow\left(\mathrm{C}-\mathrm{Cl}_{A_{m}} x \Leftrightarrow \bigwedge_{a<m} x \subset A_{a}\right)
$$

Beweis (3.3.21):

$\Rightarrow "$ : vgl. (3.3.15) „६" vgl. (3.3.16).

Beweis (3.3.22): Anwendung von (3.3.21): Für $a<m$ und $x \subset A_{a}$ gilt $\bigcup_{y \in x} \mathfrak{C} y \subset A_{a}$ (3.3.15), $\{y \mid \subset y \subset x\} \subset A_{a+1}$ (3.3.16) und trivialerweise,$y \subset x \Rightarrow y \subset A_{a}$ ".

Es bleibt noch die Relativierung des Fundamentaxioms zu zeigen und hierbei erweist es sich als nützlich, vorher die Relativierung des Fundierungsprinzips zu zeigen:

$$
\begin{gathered}
\operatorname{FUND}_{A_{m}}, \\
a<m \wedge x \subset A_{m} \wedge \mathrm{Fd}_{\boldsymbol{A}_{m}} x \subset A_{a} \Rightarrow x \subset A_{a},
\end{gathered}
$$$$
\mathrm{FD}_{A_{m}} \text {. }
$$

Beweis (3.3.23): Das Fundierungsprinzip FUND für Klassen ist bekanntlich (vgl. [81) zum Fundierungsprinzip für Mengen äquivalent, und dessen Relativierung auf $A_{m}$ gilt, weil die Mengen in $A_{m}$ genau die Mengen in den $A_{a}{ }^{\text {'s }}$ sind (3.3.8) und in den $A_{a}$ 's das Fundierungsprinzip gilt (3.2.26).

Beweis (3.3.24): Wegen (3.3.23) können wir durch $\in$-Induktion über $x$ beweisen. Für $y \in x \cap B_{m}$ ist $y \in \mathrm{Fd}_{A_{m}} x \subset A_{a}$. Und für $y \in x \backslash B_{m} \subset A_{m} \backslash B_{m} \subset \underline{M}_{\underset{\sim}{a}}((3.3 .8)$,
3.3.18)), ist $\mathrm{Fd}_{A_{m}} y \subset \mathrm{Fd}_{A_{m}} x \subset A_{a}$. Nach Induktionsvoraussetzung folgt $y \subset A_{a}$ und sogar $y \in \mathrm{M}_{a} \cap P A_{a} \subset A_{a}$ (3.3.8). Damit gilt $x \subset A_{a}$.

Beweis (3.3.25): Folgt aus (3.3.24) mit (3.3.21).

Mit (3.3.23) läßt sich dann noch zeigen, daß die Relativierung der Familie $\left(\underline{\mathrm{A}}_{a}, \underline{\mathrm{B}}_{a}\right)_{a \in \Omega}$ gerade die Familie $\left(A_{a}, B_{a}\right)_{a<m}$ ist, und aus (3.3.3) folgt dann die Gültigkeit der Relativierung des Abschlußprinzips.

$$
\mathbf{A P}_{A_{m}}
$$

Insbesondere gilt also die Relativierung des Ersetzungsaxioms für C-Klassen nicht (vgl. (2.3.15)). Insgesamt haben wir jetzt gezeigt:

Widerspruchsfreiheitstheorem 4. Die C-Theorie mit Auswahlprinziip AM für Mengen, Fundierungsprinzip FUND und Abschlußprinzip AP ist relativ widerspruchsfrei zur $\underline{M}$-Theorie mit dem Auswahlprinzip MA.

Mit dem Widerspruchsfreiheitstheorem 2 folgt somit das Widerspruchsfreiheitstheorem 3. Darüberhinaus folgt noch in der C-Theorie die Widerspruchsfreiheit des Fundierungs- únd Abschlußprinzips relativ zum Auswahlprinzip AM für Mengen. Auch hier sei noch erwähnt, daß die Widerspruchsfreiheitsresultate erhalten bleiben, wenn man die C- und M-Theorie um das starke Klassenkomprehensionsaxiom KOMPS erweitert (weil dann im Modell auch das relativierte Axiom gilt).

\section{§ 4. Die C-Theorie als Grundlage der Kategorientheorie}

Es soll jetzt erläutert werden, inwieweit die C-Theorie als mengentheoretische Grundlage der Kategorientheorie - deren Grundzüge vorausgesetzt werden, vg1. etwa [4], [10] - verwendet werden kann. Bisher wird die Kategorientheorie in der reinen NBG bzw. NBGQ Theorie oder in der Zermelo-Fraenkel'schen Mengenlehre (kurz: ZF-Theorie) mit verschieden starken „Universen-Axiomen” (vgl. Kühnrich [2]) entwickelt. Hierbei tauchen jedoch die folgenden Schwierigkeiten auf:

(1) In der NBGQ-Theorie gibt es die Kategorie $\mathscr{L}^{\mathscr{N}}$ aller Funktoren von einer Kategorie $\mathscr{K}$ in eine Kategorie $\mathscr{L}$ nur dann, wenn $\mathscr{K}$ eine kleine Kategorie ist, und es gibt keine Kategorien von nicht-kleinen Kategorien. Viele wichtige Kategorien (die aller Mengen, Gruppen etc.) sind aber nicht klein.

(2) In der ZF-Theorie mit Universen gibt es keine Kategorie aller Mengen, Gruppen etc., sondern nur ihre Relativierungen auf feste Universen. Für verschiedene Universen $\mathfrak{U}$ und $\mathfrak{B}$ sind die Kategorie der $\mathfrak{U}$-Mengen und die der $\mathfrak{B}-$ Mengen $\mathrm{im}$ allgemeinen nicht zueinander äquivalent. Außerdem steht kein globaler kategorieller Vollständigkeitsbegriff zur Verfügung (sondern nur auf Universa relativierte). 
Wir wollen daher skizzieren, wie man Kategorientheorie in der C-Theorie behandeln kann ohne auf diese Probleme zu stoßen. Hierzu betrachten wir Kategorien (im üblichen Sinn), deren Objekt- und Morphismenklasse C-Klassen sind und nennen sie C-Kategorien. Nach 2.4 ist klar, daß man zu beliebigen C-Kategorien $\mathscr{K}$ und $\mathscr{L}$ eine Funktorkategorie $\mathscr{L}^{\mathscr{K}}$ definieren kann, die wieder eine C-Kategorie ist. Nun ist die Kategorie aller Mengen (Gruppen etc.) zwar keine C-Kategorie, aber (unter Verwendung des Auswahlaxioms AM) wenigstens zu einer solche kategoriell äquivalent (vgl. 4.3 unten), und es gibt einen globalen Vollständigkeitsbegriff für Kategorien. Die C-Kategorien bilden also eine sinnvolle „Klasse” von Kategorien, und es gibt sogar die Kategorie aller C-Kategorien (die aber keine C-Kategorie mehr ist). Um darüber hinaus Kategorie von gewissen C-Kategorien bilden zu können, die wieder C-Kategorien sind, nehmen wir eine Einteilung, der C-Kategorien vor:

Für eine C-Klasse $U \subset U$ von Urelementen (bzw. eine Ordinalzahl $a \in \Omega$ ) definieren wir U-Kategorien (bzw. a-Kategorien) als diejenigen Kategorien, deren Objekte und Morphismen in $\mathrm{E}(U)$ (bzw. in $\underline{E}\left(\underline{B}_{a}\right)=\underline{A}_{a}$ ) liegen. Dann ist jede C-Kategorie $U$-Kategorie für ein geeignetes $U$ und, falls das Abschlußprinzip gilt, sogar $a$-Kategorie für ein $a \in \Omega$. Es gilt dann offenbar:

(4.0) Die Kategorie aller $U$-Kategorien ist eine C-Kategorie.

(4.1) Funktorkategorien von $a$-Kategorien sind $(a+1)$-Kategorien.

(4.2) Die Kategorie aller $a$-Kategorien ist eine $(a+1)$-Kategorie.

(4.3) ${ }^{\mathbf{A M}}$ Die Kategorie aller Mengen ist $\mathrm{zu}$ einer 0-Kategorie - die natürlich $U$ und $a$-Kategorie für jedes $U, a$ ist - äquivalent (und zwar zur vollen Teilkategorie aller Ordinalzahlen).

Hierbei wird deutlich, daß die inneren Modelle $\underline{\mathrm{E}}(U)$ bzw. $\underline{\mathrm{A}}_{a}$ im wesentlichen dieselbe Funktion der ZF-Universen in der Kategorientheorie übernehmen können ohne daß die in (2) erläuterten Nachteile auftreten. Aus dieser Sicht erscheint uns die C-Theorie als eine geeignete mengentheoretische Grundlage der Kategorientheorie.

\section{Literatur}

1] K. Gödel, The Consistency of the Axioms of Choice and of the Generalized Continum Hypothesis with the Axioms of Set Theory, Princeton 1940.

[2] M. Kühnrich, Uber den Begriff des Universums, Z. Math. Logik Grundl. Math. 12 (1966), pp. $37-50$.

[3] - Zur Definition des geordneten Paares, Z. Math. Logik Grundl. Math. 13 (1967), pp. 379-380

[4] S. Mac Lane, Categories, Berlin-Heidelberg-New York 1972.

[5] A. Oberschelp, Eigentliche Klassen als Urelemente in der Mengenlehre, Math. Annalen 157 (1964), pp. 234-260.
[6] A. Obersche1p, Set theory over classes, Preprint: Kiel 1971.

[7] W. v. O. Quine, Set theory and its logic, Cambridge (Mass.) 1963.

[8] J. E. Rubin, Set Theory for the Mathematician, San Francisco-Cambridge-London-Amsterdam 1967.

[9] J. C. Shepherdson, Inner models of set theory I-III. J. Symb. Logic 16 (1951), pp. 161-190, 17 (1952), pp. 225-237, 18 (1953), pp. 145-167.

[10] H. Schubert, Kategorien I-II, Heidelberger Taschenbücher 65-66, 1970.

111] A. Tarski, Uber Unerreichbare Kardinalzahlen, Fund. Math. 30 (1938), pp. 68-89.

FACHSEKTION MATHEMATIK, UNIVERSITÄT BREMEN Bremen

Accepté par la Rédaction le 28. 5. 1974 